Homology, Homotopy and Applications, vol.10(2), 2008, pp.13-58

\title{
THE ALGEBRAIC $K$-THEORY OF A DIAGRAM OF RINGS
}

\author{
JEANNE DUFLOT \\ (communicated by Charles Weibel)
}

\begin{abstract}
In this paper, we consider "diagrams of rings", or functors from a small category to the category of rings, and the corresponding diagrams of groups $K_{i}$. Classically, this was initiated by Milnor [13]. The main result of this paper is the direct comparison of the filtration in classical algebraic $K$-theory discussed in $[6,7]$ to a corresponding filtration in the BousfieldKan spectral sequence associated to a Tot-tower of simplicial groups attached to the diagram of rings.
\end{abstract}

\section{Introduction}

This paper is the initial part of a study of the Bousfield-Kan spectral sequence associated to certain types of cosimplicial simplicial groups and its relationship to the work presented in $[\mathbf{6}, \mathbf{7}]$. These relationships are primarily developed in Sections 5 and 6 . Much of the remaining sections is expository. Below is an outline of the paper.

- Sections 2.1-2.3 present notation, definitions and basic results from the literature. Section 2.4 presents a particular model for computing the homotopy of $\operatorname{Tot}_{1} G$, for a cosimplicial simplicial group $G$, which is used in the subsequent work. Section 2.5 returns to summarize homotopy properties of the constructions used in the paper.

- Section 3 presents a review of the details of the Bousfield-Kan spectral sequence, discussing particular properties that it possesses when applied to the types of cosimplicial simplicial groups discussed in the subsequent work. The general construction of the Bousfield-Kan spectral sequence [4] for any cosimplicial simplicial group $X$ is first outlined and an extension of the spectral sequence is constructed for the types of cosimplicial simplicial groups considered in the applications, remedying the defect of "fringedness" that the general spectral sequence possesses. When the cosimplicial simplicial group $X$ satisfies the "cofiniteness" condition of vanishing $\pi_{*}\left(N X^{s}\right)$ for large $s$, the spectral sequence converges strongly to the homotopy groups of $\operatorname{Tot}(X)$. The purpose of this is to give an exposition of a (known) remedy for the defects of the Bousfield-Kan spec-

Received February 22, 2007, revised April 18, 2008; published on July 22, 2008.

2000 Mathematics Subject Classification: 55U10, 18G30, 18G55, 19 D99.

Key words and phrases: algebraic $K$-theory, simplicial group.

This article is available at http://intlpress.com/HHA/v10/n2/a2

Copyright (C) 2008, International Press. Permission to copy for private use granted. 
tral sequence, found not by passing to the category of spectra but, instead, by restricting to an appropriate category of cosimplicial simplicial groups.

- Sections 4 through 5.2 provide a review of notation, definitions and results from $[\mathbf{6}, \mathbf{7}]$. In [7], if $R$ is the inverse limit of a diagram of rings $\mathfrak{R}$ over a small category $\mathcal{I}$, then a filtration

$$
\mathcal{F}_{2} \subseteq \mathcal{F}_{1} \subseteq \mathcal{F}_{0} \subseteq K_{0}(R)
$$

is constructed on the classical Grothendieck group $K_{0}(R)$. This filtration has successive quotients related to the cohomology groups $H^{i}\left(\mathcal{I}, K_{i} \mathfrak{R}\right)$, for $i=0,1,2$, and is defined in terms of certain long exact sequences in nonabelian cohomology.

In [6], given a ring $R$, a particular model for a simplicial group $G R$, whose homotopy groups yield the algebraic $K$-theory of the ring, is considered, and explicit natural identifications $\xi: K_{i}(R) \rightarrow \pi_{i} G R$ are constructed, where $K_{i}(R)$ are the classical $K$-groups of $R$, for $i=0,1$.

- In Section 5.3, the constructions of Sections 3 through 5.2 are compared, resulting in:

Theorem 1.1. There is a homomorphism

$$
j: K_{0}(\underbrace{\lim }_{\mathcal{I}} \mathfrak{R}) \rightarrow \pi_{0}(\operatorname{Tot}(\mathcal{I}, G \Re))
$$

(induced by $\xi$ ), and, the inverse image of the filtration given by the extended Bousfield-Kan spectral sequence

$$
F_{1,0} \subseteq F_{0,0} \subseteq \pi_{0}(\operatorname{Tot}(\mathcal{I}, G \Re))
$$

under the homomorphism $j$ contains the filtration

$$
\mathcal{F}_{1} \subseteq \mathcal{F}_{0} \subseteq K_{0}(\underbrace{\lim }_{\mathcal{I}} \Re)
$$

- In Section 6, the "Mayer-Vietoris" sequence of classical $K$-theory [13] is viewed via the constructions of this paper. The main result of this section is

Lemma 1.2. Given a diagram $\mathfrak{R}$ of rings

$$
\begin{array}{ccc} 
& & R_{1} \\
& & \downarrow f \\
R_{2} \stackrel{g}{\rightarrow} & R_{12},
\end{array}
$$

yielding a diagram of simplicial groups $G \Re$

$$
\begin{array}{ccc} 
& G R_{1} \\
& \downarrow G f \\
G R_{2} \quad \stackrel{G g}{\rightarrow} \quad G R_{12},
\end{array}
$$

such that $g$ is surjective and $G g$ is a fibration, there is a commuting square

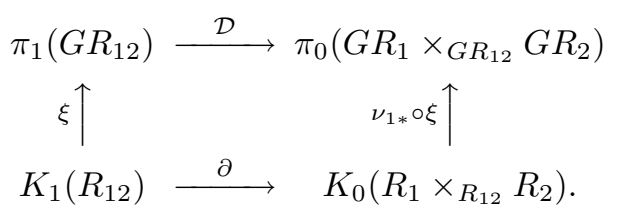


The bottom arrow in the diagram of the lemma is the connecting homomorphism of Milnor [13] constructed in classical $K$-theory, and the top arrow is one coming from the spectral sequence for the simple example of a pullback diagram of rings.

\section{Preliminaries}

For the definitions of cosimplicial spaces (i.e., cosimplicial simplicial sets) cosimplicial simplicial groups, simplicial groups and cosimplicial groups, see $[\mathbf{4 ,}, \mathbf{8 , 1 2}]$ or $[\mathbf{1 5}]$. The face, degeneracy, coface and codegeneracy maps will be denoted by using upper and lower indices: $d_{i}, s_{j}, d^{k}, s^{l}$.

In the category of simplicial groups, all homotopy groups will be taken using the identity element as a basepoint.

\subsection{The homotopy groups of a simplicial group}

If $G$ is a simplicial group, then one can compute the homotopy groups of $G$ in the following way $([\mathbf{1 2}])$ : for each $t \geqslant 1$, define

$$
\bar{G}_{t}=G_{t} \cap \operatorname{ker} d_{0} \cap \cdots \cap \operatorname{ker} d_{t-1}
$$

if $t=0$,

$$
\bar{G}_{0}=G_{0}
$$

Then (Proposition 17.3 from [12]):

(i) $d_{t+1}\left(\bar{G}_{t+1}\right) \doteq B G_{t} \subseteq \bar{G}_{t}, t \geqslant 0$.

(ii) $B G_{t} \subseteq Z G_{t} \doteq \operatorname{ker} d_{t}: \bar{G}_{t} \rightarrow \bar{G}_{t-1}, t>0$.

(iii) $B G_{t}$ is a normal subgroup of both $\bar{G}_{t}$ and of $G_{t}$.

Thus,

$$
\cdots \stackrel{d_{t+2}}{\longrightarrow} \bar{G}_{t+1} \stackrel{d_{t+1}}{\longrightarrow} \bar{G}_{t} \stackrel{d_{t}}{\longrightarrow} \bar{G}_{t-1} \stackrel{d_{t-1}}{\longrightarrow} \cdots \rightarrow
$$

becomes a chain complex, generally nonabelian. Then one proves that the "homology" groups of this chain complex are the homotopy groups of the simplicial group $G$. Note that $\pi_{t}(G)$ is an abelian group $([\mathbf{1 2}])$ if $t \geqslant 1$, and that $\pi_{0}(G)$ is a group. The product in $\pi_{t}(G)$ may be computed in the following way $([\mathbf{1 2}])$ : if $\left[g_{1}\right],\left[g_{2}\right]$ are in $\pi_{t}(G)$ (where $[g]$ denotes the coset of the element $g$ ), then $\left[g_{1}\right]\left[g_{2}\right]=\left[g_{1} g_{2}\right]$.

One can prove the following well-known lemma [15]:

Lemma 2.1. Let $f: G \rightarrow H$ be a homomorphism of simplicial groups that is also a fibration. Define the simplicial group $F \doteq \operatorname{ker} f$; face maps and degeneracy maps are of course those coming from $G$. Then

(a) For every $t \geqslant 1$, the induced homomorphism $f: \bar{G}_{t} \rightarrow \bar{H}_{t}$ is surjective.

(b) The following is a diagram of exact sequences (NB: if $t=1$ the bottom sequence is not necessarily short exact in that the end arrow may not be surjective!):

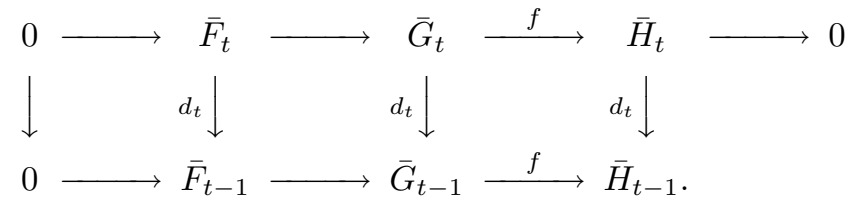


(c) The long exact sequence in homotopy,

$$
\cdots \stackrel{\delta}{\rightarrow} \pi_{t}(F) \rightarrow \pi_{t}(G) \stackrel{f_{*}}{\rightarrow} \pi_{t}(H) \stackrel{\delta}{\rightarrow} \pi_{t-1}(F) \rightarrow \cdots
$$

may be constructed with $\delta$ defined in the following way: Let $[h] \in \pi_{t}(H)(t \geqslant 1)$. Then there exists an element $g \in \bar{G}_{t}$ such that $f(g)=h$. Also,

$$
d_{t}(g) \in \bar{F}_{t-1} \cap \operatorname{ker} d_{t-1} .
$$

Define $\delta([h])=\left[d_{t}(g)\right]$.

Moreover, all the maps in this long exact sequence are group homomorphisms, and $\operatorname{im}\left(\delta: \pi_{1}(H) \rightarrow \pi_{0}(F)\right)$ is a central subgroup of $\pi_{0}(F)$.

(d) Conversely: if $\tilde{f}$ is any homomorphism of simplicial groups such that for every $t \geqslant 1$, the induced homomorphism $\tilde{f}: \bar{G}_{t} \rightarrow \bar{H}_{t}$ is surjective, then indeed $\tilde{f}$ is a fibration.

Consider the following commutative diagram of simplicial groups, where every row and column are fibration sequences (the maps $p$ and $q$ are fibrations, and we will regard $i, j$ as inclusion maps of the kernels of the homomorphisms $t$ and $p$ respectively):

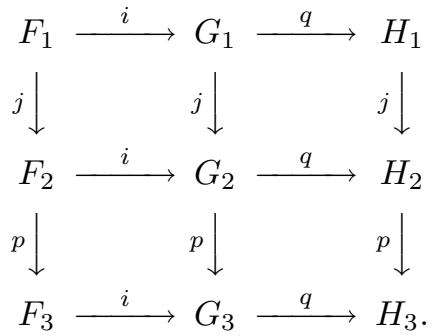

Then, there is a square (for every $t \geqslant 1$ ) for two connecting homomorphisms $\delta, \Delta$ defined as in the previous lemma:

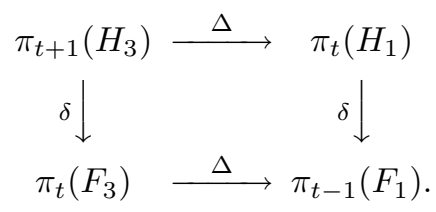

We will need the following (also well-known) lemma.

Lemma 2.2. With $\Delta, \delta$ defined as in the previous lemma, in the above diagram

$$
(\Delta \circ \delta)(x)=(\delta \circ \Delta)(x)^{-1},
$$

for every $x \in \pi_{t+1}\left(H_{3}\right)$, and every $t \geqslant 1$.

In this instance, we switch to additive notation, defining $-\Delta$ to be the homomorphism such that $-\Delta(x)=\Delta(x)^{-1}$ for every $x$ in the domain of $\Delta$. Note that $-\Delta$ is indeed a homomorphism, since the domain of $\Delta$ is always an abelian group. 


\subsection{The cohomotopy groups of a cosimplicial group}

Let $G$ be a cosimplicial group. The normalized groups $N G^{*}$ are defined by

$$
N G^{m}=\bigcap_{j=0}^{m-1} \operatorname{ker}\left(s^{j}: G^{m} \rightarrow G^{m-1}\right)
$$

for $m \geqslant 1$. The group $N G^{0}$ is defined to be $G^{0}$.

If $\mu: G \rightarrow H$ is a homomorphism of cosimplicial groups, then we denote the induced homomorphisms from $N G^{m} \rightarrow N H^{m}$ by $\mu$ as well.

The zero-th cohomotopy group of $G$ is

$$
\pi^{0}(G)=\left\{g \in N G^{0} \mid d^{0}(g)=d^{1}(g)\right\} .
$$

If $\mu: G \rightarrow H$ is a homomorphism of cosimplicial groups, then the restriction of $\mu$ to $\pi^{0}(G)$ will be denoted by $\mu_{*}$.

The first cohomotopy set is defined as follows:

Let

$$
Z^{1}(G)=\left\{g \in N G^{1} \mid d^{1}(g)=d^{2}(g) d^{0}(g)\right\} .
$$

$Z^{1}$ is not necessarily a subgroup of $N G^{1}$, but it does have the identity element 1 of $G^{1}$ in it, and is thus considered as a pointed set with basepoint equal to 1 .

Now, the group $N G^{0}$ acts on the set $Z^{1}(G)$ on the right as follows: If $g \in N G^{0}$ and $f \in Z^{1}(G)$, then

$$
f \cdot g=d^{1}(g)^{-1} f d^{0}(g) .
$$

Define $\pi^{1}(G)$ to be the set of orbits of the action of $N G^{0}$ on $Z^{1}(G)$. The set $\pi^{1}(G)$ is a pointed set with basepoint equal to the orbit of 1 . Notice that the orbit of 1 is a set in one-to-one correspondence with the coset space $N G^{0} / \pi^{0}(G)$.

If $\mu: G \rightarrow H$ is a homomorphism of cosimplicial groups, then $\mu$ takes $Z^{1}(G)$ to $Z^{1}(H)$ since it commutes with the operators $d^{i}$ and $s^{0}$. Since the actions of $N G^{0}$ and $N H^{0}$ on $Z^{1}(G)$ and $Z^{1}(H)$ are defined in terms of the operators $d^{i}$ as well, these actions are equivariant with respect to $\mu$, and thus there is a basepoint preserving induced function $\mu_{*}: \pi^{1}(G) \rightarrow \pi^{1}(H)$.

As far as the higher cohomotopy groups go, we shall only be interested in these if $G$ is an abelian cosimplicial group.

So, in case $G$ is an abelian cosimplicial group, $\pi^{1}(G)$ is also an abelian group (with multiplication induced by that of $G$ ) and one may also define higher cohomotopy groups $\pi^{i}(G)$ for $i \geqslant 2$; let

$$
Z^{i}(G)=\left\{g \in N G^{i} \mid \prod_{j=0}^{i+1} d^{j}(g)^{(-1)^{j}}=1\right\}
$$

and let

$$
B^{i}(G)=\left\{g \in N G^{i} \mid \exists h \in N G^{i-1} \ni g=\prod_{j=0}^{i} d^{j}(h)^{(-1)^{j}}\right\} .
$$

Then, $B^{i}(G)$ is a subgroup of $Z^{i}(G)$ and we define

$$
\pi^{i}(G)=Z^{i}(G) / B^{i}(G) .
$$


Naturally, one has induced homomorphisms $\mu_{*}: \pi^{i}(G) \rightarrow \pi^{i}(H)$ for every map of cosimplicial groups $\mu: G \rightarrow H$.

\subsection{Constructions from homotopy theory}

We give some details of the basic definitions used in the construction of the Bousfield-Kan spectral sequence. References for this section are $[4,8]$.

Let $\Delta$ be the cosimplicial space formed by the standard simplices. In other words,

$$
\Delta_{j}^{i}=\{\alpha: \mathbf{j} \rightarrow \mathbf{i} \mid \alpha \text { is nondecreasing }\} \doteq[\mathbf{j}, \mathbf{i}] ;
$$

here, if $n \geqslant 0$ is a nonnegative integer, then

$$
\mathbf{n}=\{0,1, \ldots, n\}
$$

is the set of integers between 0 and $n$ ordered in the usual way.

The simplicial and cosimplicial structure on $\Delta$ is as follows. If $\tau: \mathbf{i}_{\mathbf{1}} \rightarrow \mathbf{i}$ is a nondecreasing map, then

$$
\tau_{*}: \Delta_{j}^{i_{1}} \rightarrow \Delta_{j}^{i}
$$

is the function

$$
\tau_{*}(\alpha)=\tau \circ \alpha .
$$

If $\sigma: \mathbf{j}_{\mathbf{1}} \rightarrow \mathbf{j}$ is a nondecreasing map, then

$$
\sigma^{*}: \Delta_{j}^{i} \rightarrow \Delta_{j_{1}}^{i}
$$

is the function

$$
\sigma^{*}(\alpha)=\alpha \circ \sigma .
$$

If $q \geqslant 0$, then the $q$-skeleton of $\Delta$ is the cosimplicial space defined by

$$
S k_{q}(\Delta)_{j}^{n}=\{\alpha \in[\mathbf{j}, \mathbf{n}] \mid \exists r \leqslant q, \exists \beta \in[\mathbf{r}, \mathbf{n}], \exists \gamma \in[\mathbf{j}, \mathbf{r}] \text { э } \alpha=\beta \circ \gamma\} ;
$$

the cosimplicial structure is that coming from $\Delta$. In other words, $S k_{q}(\Delta)$ is the subspace of $\Delta$ generated by the simplices of dimension less than or equal to $q$.

Now, if $n$ and $q$ are fixed nonnegative integers, then we may form the cosimplicial spaces $\Delta^{n} \times \Delta$ and $\Delta^{n} \times S k_{q}(\Delta)$, defined by

$$
\left(\Delta^{n} \times \Delta\right)_{j}^{i}=\Delta_{j}^{n} \times \Delta_{j}^{i},\left(\Delta^{n} \times S k_{q}(\Delta)\right)_{j}^{i}=\Delta_{j}^{n} \times S k_{q}(\Delta)_{j}^{i} .
$$

The cosimplicial space structure is described by:

If $\theta: \mathbf{i}_{\mathbf{1}} \rightarrow \mathbf{i}_{\mathbf{2}}$ is a nondecreasing map, then

$$
\theta_{*}: \Delta_{j}^{n} \times \Delta_{j}^{i_{1}} \rightarrow \Delta_{j}^{n} \times \Delta_{j}^{i_{2}}
$$

is the function

$$
\theta_{*}(\alpha, \beta)=(\alpha, \theta \circ \beta),
$$

for every $j$. 
If $\tau: \mathbf{j}_{\mathbf{1}} \rightarrow \mathbf{j}_{\mathbf{2}}$ is a nondecreasing map, then

$$
\tau^{*}: \Delta_{j_{2}}^{n} \times \Delta_{j_{2}}^{i} \rightarrow \Delta_{j_{1}}^{n} \times \Delta_{j_{1}}^{i}
$$

is the function

$$
\tau^{*}(\alpha, \beta)=(\alpha \circ \tau, \beta \circ \tau)
$$

for every $i$.

\subsubsection{Paths and loops}

The first constructions we are interested in are the path and loop constructions. We outline two models for these constructions; one model is that used for any fibrant simplicial set, and the second is one used only for simplicial groups.

If $G$ is a simplicial group, it is fibrant as a pointed simplicial set [12]. Thus we can form the usual path and loop space constructions, defined, in fact, for any fibrant simplicial set, outlined in the following.

First, for $\epsilon=0,1$, form the simplicial sets $\operatorname{Hom}_{\mathcal{S}}\left(\Delta^{\epsilon}, G\right)$, whose set of $n$-simplices is by definition

$$
\operatorname{Hom}_{\mathcal{S}}\left(\Delta^{\epsilon}, G\right)_{n}=\operatorname{hom}_{\mathcal{S}}\left(\Delta^{n} \times \Delta^{\epsilon}, G\right)
$$

where the right-hand hom-set is the set of maps of simplicial sets (the category of simplicial sets is denoted by $\mathcal{S}$ ).

These simplicial sets are simplicial groups under pointwise multiplication of functions.

Then, $\mathbf{P} G$ is defined to be the kernel of the homomorphism of simplicial groups

$$
\operatorname{Hom}_{\mathcal{S}}\left(\Delta^{1}, G\right) \stackrel{\left(d^{0}\right)^{*}}{\rightarrow} \operatorname{Hom}_{\mathcal{S}}\left(\Delta^{0}, G\right) \cong G .
$$

If $d^{0}, d^{1}: \Delta^{0} \rightarrow \Delta^{1}$ are the standard coface maps, then the maps $\left(d^{0}\right)^{*}$ and $\left(d^{1}\right)^{*}$ from $\operatorname{Hom}_{\mathcal{S}}\left(\Delta^{1}, G\right)$ to $\operatorname{Hom}_{\mathcal{S}}\left(\Delta^{0}, G\right)$ are defined by $\left(d^{\epsilon}\right)^{*}(g)=g \circ\left(1 \times d^{\epsilon}\right)$, for $\epsilon=0$ or 1 .

The homomorphism of simplicial groups

$$
\pi: \mathbf{P} G \rightarrow G
$$

is defined to be the composite

$$
\mathbf{P} G \hookrightarrow \operatorname{Hom}_{\mathcal{S}}\left(\Delta^{1}, G\right) \stackrel{\left(d^{1}\right)^{*}}{\rightarrow} \operatorname{Hom}_{\mathcal{S}}\left(\Delta^{0}, G\right) \cong G .
$$

The loop space $\boldsymbol{\Omega} G$ is the kernel of $\pi$. As is well known ([8]), $\pi$ is a fibration (of simplicial groups) whose total space $\mathbf{P} G$ is weakly equivalent to a point. The fibration sequence

$$
\Omega G \rightarrow \mathbf{P} G \rightarrow G
$$

is functorial in $G$ as well. 
The long exact sequence of homotopy groups associated to the fibration sequence

$$
\mathbf{\Omega} \rightarrow \mathbf{P} G \rightarrow G
$$

yields a natural isomorphism of groups

$$
\Delta: \pi_{i+1}(G) \rightarrow \pi_{i}(\boldsymbol{\Omega} G)
$$

for every $i \geqslant 0$, since $\mathbf{P} G$ is weakly equivalent to a point.

If $H$ is a cosimplicial simplicial group, then for each $i \geqslant 0, H^{i}$ is a simplicial group and the cofaces and codegeneracies are homomorphisms of simplicial groups $H^{\alpha} \rightarrow H^{\beta}$ for appropriate $\alpha$ and $\beta$. Thus, we may form the cosimplicial simplicial groups $\mathbf{P} H$ obtained by using the construction $\mathbf{P}$ of the above paragraph on each simplicial group $H^{i}$; the coface and codegeneracy maps are obtained from applying the functor $\mathbf{P}$ to the cofaces and codegeneracies of $H$. Then, the homomorphisms $\pi$ induce a homomorphism $\pi: \mathbf{P} H \rightarrow H$ of cosimplicial simplicial groups. The kernel of this homomorphism is exactly the cosimplicial simplicial group $\Omega H$ obtained by using the construction $\boldsymbol{\Omega}$ of the above paragraph on each simplicial group $H^{i}$, with the coface and codegeneracy maps obtained from applying the functor $\Omega$ to the cofaces and codegeneracies of $H$.

The sequence $\Omega H \rightarrow \mathbf{P} H \rightarrow H$ is a fibration sequence in the category of cosimplicial simplicial groups as well.

Another model for the fibration sequence $\Omega G \rightarrow P G \rightarrow G$ for a simplicial group $G$ is constructed as follows. We use $[\mathbf{9}]$ as a reference.

The simplicial group $G^{I}$ is defined by

$$
\left(G^{I}\right)_{q}=\left\{\left(x_{0}, \ldots, x_{q}\right) \in G_{q+1} \times \cdots \times G_{q+1} \mid d_{i} x_{i}=d_{i} x_{i-1}, 1 \leqslant i \leqslant q\right\}
$$

with face operators

$$
d_{i}\left(x_{0}, \ldots, x_{q}\right)=\left(d_{i+1} x_{0}, \ldots, d_{i+1} x_{i-1}, d_{i} x_{i+1}, \ldots, d_{i} x_{q}\right)
$$

The two maps of simplicial groups, for $\epsilon=0,1$

$$
\partial_{\epsilon}: G^{I} \rightarrow G
$$

defined by

$$
\partial_{\epsilon}\left(x_{0}, \ldots, x_{q}\right)= \begin{cases}d_{0} x_{0}, & \epsilon=0 \\ d_{q+1} x_{q}, & \epsilon=1\end{cases}
$$

are fibrations. $P G$ is here defined as ker $\partial_{0}$. Also, the map $\left.\partial_{1}\right|_{P G}: P G \rightarrow G$ is a fibration, and $\Omega G$ is defined as the kernel of this homomorphism. Then one has (for a basic reference see $[\mathbf{1 2}$, Section 6$])$ :

Lemma 2.3. There is an isomorphism $\phi: \operatorname{Hom}\left(\Delta^{1}, G\right) \rightarrow G^{I}$ of simplicial groups, defined by

$$
\phi(\tilde{c})=\left(\ldots, \tilde{c}\left(s^{k}, s^{0} \cdots \hat{s}^{k} \cdots s^{i}\right), \ldots\right)
$$


if $\tilde{c}$ is of degree $i$. This isomorphism defines an isomorphism of fibrations of simplicial groups

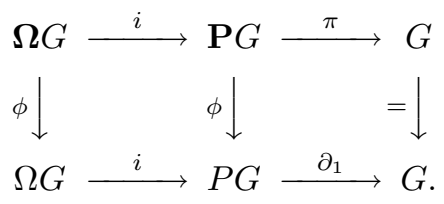

We will use both models in this paper.

\subsubsection{Loopedness}

If $G$ is a cosimplicial simplicial group, and if $i \geqslant 0$ is a fixed (cosimplicial) index, then there is a simplicial group $N G^{i}$ formed by the set of groups $\left\{N G_{j}^{i} \mid j \geqslant 0\right\}$. If $j \geqslant 0$ is a fixed (simplicial) index, then there is a cosimplicial group $\left\{\pi_{j}\left(G^{i}\right) \mid i \geqslant 0\right\}$, which is a subquotient of the cosimplicial group $G_{j}$, with cofaces and codegeneracies induced by those of $G$.

We will say that a cosimplicial simplicial group $G$ is "looped" if and only if there is a cosimplicial simplicial group $H$, and a homomorphism of cosimplicial simplicial groups $\theta: G \rightarrow \boldsymbol{\Omega} H$ such that $\theta$ induces an isomorphism of cosimplicial groups $\pi_{j}(G) \rightarrow \pi_{j}(\boldsymbol{\Omega} H)$ for every every $j \geqslant 0$. We will say that such a $\theta$ is a "simplicial-wise weak equivalence" of cosimplicial simplicial groups.

There is a suitable category of looped cosimplicial simplicial groups: the objects are all triples $(G, \theta, H)$, where $G$ and $H$ are cosimplicial simplicial groups, and $\theta: G \rightarrow \boldsymbol{\Omega} H$ is a simplicial-wise weak equivalence; the morphisms are "maps of triples". We leave this to the reader to formally write down, but note that the maps $G \rightarrow \tilde{G}$ and $H \rightarrow \tilde{H}$ comprising a morphism should be homomorphisms of cosimplicial simplicial groups.

Similarly, one can define a "looped" simplicial group, and a category of looped simplicial groups.

A looped cosimplicial simplicial group has the property that for every $j \geqslant 0$, the cosimplicial groups $\pi_{j}(G)$ are abelian cosimplicial groups: for $j \geqslant 2$, this is of course true for any cosimplicial simplicial set; for $j=1$, this is because we have a cosimplicial simplicial group; for $j=0$, this is because we have a looped cosimplicial simplicial group.

We are interested in the following sort of cosimplicial simplicial group $G$ :

- $G$ is looped: there is a cosimplicial simplicial group $H$ and a simplicial-wise weak equivalence $\theta: G \rightarrow \boldsymbol{\Omega} H$ that is also a homomorphism of cosimplicial simplicial groups.

- There exists an integer $M \geqslant 0$ such that the homotopy groups $\pi_{j}\left(N H^{i}\right)$ of the simplicial groups $N H^{i}$ are equal to the identity group, for every $i>M$ and every $j \geqslant 0$.

In the rest of this paper, we will call such a (cosimplicial simplicial) group a "cofinite looped (cosimplicial simplicial) group".

\subsubsection{Tot and Tot $_{s}$}

The next constructions we review are those of the simplicial groups $\operatorname{Tot}_{q} G$ functorially associated to a cosimplicial simplicial group $G$ (More generally, the Tot constructions 
may be made for any cosimplicial space.)

For each $q \geqslant 0$, the simplicial group $\operatorname{Tot}_{q} G$ is defined by specifying its $n$-simplices as

$$
\left(\operatorname{Tot}_{q} G\right)_{n}=\operatorname{hom}_{c \mathcal{S}}\left(\Delta^{n} \times S k_{q}(\Delta), G\right) \doteq\left(\operatorname{Hom}_{c \mathcal{S}}\left(S k_{q}(\Delta), G\right)\right)_{n}
$$

in other words, $\left(\operatorname{Tot}_{q} G\right)_{n}$ is the set of cosimplicial space maps from $\Delta^{n} \times S k_{q}(\Delta)$ to $G$ (we denote the category of cosimplicial simplicial sets by $c \mathcal{S}$ ). This set is a group with respect to pointwise multiplication. If $q<0$, define $\operatorname{Tot}_{q} G$ to be the trivial simplicial group.

The simplicial set structure on $\operatorname{Tot}_{q} G$ is described by:

If $\theta \in\left[\mathbf{n}_{1}, \mathbf{n}_{2}\right]$, then $\theta^{*}:\left(\operatorname{Tot}_{q} G\right)_{n_{2}} \rightarrow\left(\operatorname{Tot}_{q} G\right)_{n_{1}}$ is

$$
\theta^{*}(f)(\alpha, \beta)=f(\theta \circ \alpha, \beta),
$$

for $(\alpha, \beta) \in \Delta^{n} \times S k_{q}(\Delta)$. These maps are all group homomorphisms, giving $\operatorname{Tot}_{q} G$ the structure of a simplicial group.

Note that one has inclusions of cosimplicial spaces

$$
\cdots \subset S k_{q-1}(\Delta) \subset S k_{q}(\Delta) \subset \cdots \subset \Delta
$$

and

$$
\Delta=\bigcup_{q \geqslant 0} S k_{q}(\Delta) .
$$

The above inclusions induce simplicial group homomorphisms

$$
\cdots \stackrel{p}{\rightarrow} \operatorname{Tot}_{q} G \stackrel{p}{\rightarrow} \operatorname{Tot}_{q-1} G \stackrel{p}{\rightarrow} \cdots \stackrel{p}{\rightarrow} \operatorname{Tot}_{0} G .
$$

We will refer to this tower as the Tot-tower for the simplicial group $G$. It is functorial in $G$.

According to [4] these maps $p$ are all fibrations of simplicial sets; thus, being homomorphisms of simplicial groups, they are all also fibrations in the closed model category of simplicial groups.

The simplicial set $\operatorname{Tot}(G)$ can be defined to be the inverse limit of the above tower of fibrations. One may describe $\operatorname{Tot}(G)$ as the simplicial set whose $n$-simplices is the set of cosimplicial space maps from $\Delta^{n} \times \Delta$ to $G$.

This set is a group with respect to pointwise multiplication. There are natural homomorphisms of simplicial groups (which are also fibrations) $\tilde{p}: \operatorname{Tot}(G) \rightarrow \operatorname{Tot}_{q} G$, for every $q$, giving commuting triangles with the maps $p: \operatorname{Tot}_{q} G \rightarrow \operatorname{Tot}_{q-1} G$. Note that $\operatorname{Tot}(G)$, being a group, is always nonempty: the identity element (which is the constant map at $1 \in G)$ is certainly in $\operatorname{Tot}(G)$.

One can compute that the simplicial groups $\operatorname{Tot}_{0} G$ and $G^{0}$ are isomorphic simplicial groups.

The description of Tot $_{q}$ above is not so useful in computations; more useful is the principle below, enunciated in [8], describing how Tot $_{q}$ is built from Tot $_{q-1}$, using a pullback diagram of simplicial groups (where $C$ is a cosimplicial simplicial group):

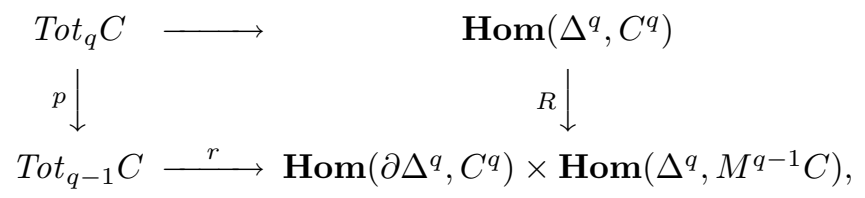


where the simplicial group $M^{q-1} C$ is defined for $q=0,1$ by

$$
M^{q-1} C= \begin{cases}1, & q=0 \\ C^{0}, & q=1\end{cases}
$$

and, if $q>1$, as the equalizer of the diagram

$$
\prod_{i=0}^{q-1} C^{q-1} \underset{B}{\stackrel{A}{\rightrightarrows}} \prod_{0 \leqslant i<j \leqslant q-1} C^{q-2} .
$$

Here, $A$ and $B$ are defined by (the lower double subscript denotes that particular component of the indicated element of the product)

$$
\begin{aligned}
& A\left(y_{0}, \ldots, y_{q-1}\right)_{i j}=s^{i} y_{j}, \\
& B\left(y_{0}, \ldots, y_{q-1}\right)_{i j}=s^{j-1} y_{i} .
\end{aligned}
$$

We describe the maps $r$ and $R$ below.

To set notation, if $g: X \rightarrow Y$ is any map of cosimplicial spaces (i.e., cosimplicial simplicial sets), then for every cosimplicial index $a$, the map $g^{a}: X^{a} \rightarrow Y^{a}$ is the map of simplicial sets induced by $g$, and, for every simplicial index $b$, the map $g_{b}: X_{b} \rightarrow Y_{b}$ is the map of cosimplicial sets induced by $g$.

An element $f$ of $\operatorname{Tot}_{q-1} C$, let us say of degree $t$, is a map of cosimplicial spaces $\Delta^{t} \times S k_{q-1} \Delta \rightarrow C$. Then, $f^{q}$ is a map of simplicial sets $\Delta^{t} \times\left(S k_{q-1} \Delta\right)^{q} \rightarrow C^{q}$; thus it gives a map of simplicial sets $\Delta^{t} \times \partial \Delta^{q} \rightarrow C^{q}$; i.e., $f^{q}$ defines a degree $t$ element of $\operatorname{Hom}\left(\partial \Delta^{q}, C^{q}\right)$. Also, $f^{q-1}$ is a map of simplicial sets $\Delta^{t} \times \Delta^{q-1} \rightarrow C^{q-1}$. Since $s^{i}: \Delta^{q} \rightarrow \Delta^{q-1}$ for $0 \leqslant i \leqslant q-1$, the map $f^{q-1} \circ\left(1 \times s^{i}\right)$ is a simplicial map $\Delta^{t} \times \Delta^{q} \rightarrow C^{q-1}$, for $0 \leqslant i \leqslant q-1$. Then, the map $r$ is defined (in degree $t$ ) by

$$
f \mapsto\left(f^{q},\left(f^{q-1} \circ\left(1 \times s^{0}\right), \ldots, f^{q-1} \circ\left(1 \times s^{q-1}\right)\right)\right) .
$$

There is a homomorphism of simplicial groups

$$
\theta: C^{q} \rightarrow M^{q-1} C
$$

given by

$$
z \mapsto\left(s^{0} z, \ldots, s^{q-1} z\right) .
$$

Then, the map $R$ is defined by

$$
h \mapsto\left(\left.h\right|_{\partial \Delta^{q}}, \theta \circ h\right),
$$

for $h \in \operatorname{Hom}\left(\Delta^{q}, C^{q}\right)$.

\subsection{A computational model for $\overline{T o t}_{1}$}

We may consider $\overline{\operatorname{Tot}_{q} C}$ as a nonabelian chain complex as in Section 2.1, for every $q \geqslant 0$.

We have already seen that $\operatorname{Tot}_{0} C$ is identified with $C^{0}$; thus the chain complex $\overline{T o t}_{0}$ is identified with the chain complex $\bar{C}^{0}$. 
2.4.1. Tot $_{1}$

We may compute $\operatorname{Tot}_{1} C$ as the pullback of the diagram

$$
\begin{gathered}
\operatorname{Hom}\left(\Delta^{1}, C^{1}\right) \\
\downarrow \\
C^{0} \rightarrow \quad C^{1} \times C^{1} \times \operatorname{Hom}\left(\Delta^{1}, C^{0}\right),
\end{gathered}
$$

where the horizontal arrow is the homomorphism

$$
c \mapsto\left(d^{0} c, d^{1} c, c \circ\left(1 \times s^{0}\right)\right)
$$

and the vertical arrow is the homomorphism

$$
g \mapsto\left(g \circ\left(1 \times d^{0}\right), g \circ\left(1 \times d^{1}\right), s^{0} \circ g\right) .
$$

Here, $d^{0}, d^{1}: \Delta^{0} \rightarrow \Delta^{1}$ or $C^{0} \rightarrow C^{1} ; s^{0}: C^{1} \rightarrow C^{0}$ or $\Delta^{1} \rightarrow \Delta^{0}$. Also, we are identifying an element $x \in C_{t}^{s}$ with a simplicial set map $x: \Delta^{t} \rightarrow C^{s}$, for any $s, t$. Using $\Delta^{t} \times \Delta^{0}=\Delta^{t}$ for any $t$ we see that the map $1 \times s^{0}: \Delta^{t} \times \Delta^{1} \rightarrow \Delta^{t} \times \Delta^{0}$ may be identified with projection on the first factor $\Delta^{t} \times \Delta^{1} \rightarrow \Delta^{t}$, for any $t$.

In other words, $\operatorname{Tot}_{1} C$ is the subgroup of the simplicial group $C^{0} \times \operatorname{Hom}\left(\Delta^{1}, C^{1}\right)$ defined by

$$
\begin{aligned}
\operatorname{Tot}_{1} C & =\left\{(c, \tilde{c}) \in C^{0} \times \operatorname{Hom}\left(\Delta^{1}, C^{1}\right) \mid\left(d^{0} c, d^{1} c, c \circ\left(1 \times s^{0}\right)\right)\right. \\
& \left.=\left(\tilde{c} \circ\left(1 \times d^{0}\right), \tilde{c} \circ\left(1 \times d^{1}\right), s^{0} \circ \tilde{c}\right)\right\},
\end{aligned}
$$

and the map $\operatorname{Tot}_{1} \rightarrow \operatorname{Tot}_{0}$ sends $(c, \tilde{c})$ to $c$. Writing out the conditions for $(c, \tilde{c})$ to be an element of $\left(\operatorname{Tot}_{1} C\right)_{t}$ explicitly, for $(a, y) \in \Delta^{t} \times \Delta^{0}, \tilde{c}\left(a, d^{\epsilon} y\right)=a^{*} d^{\epsilon} c$, if $\epsilon$ is 0 or 1 ; and for $(a, b) \in \Delta^{t} \times \Delta^{1}, a^{*} c=s^{0}(\tilde{c}(a, b))$.

In addition, the face maps $d_{j}:\left(\operatorname{Tot}_{1}\right)_{*} \rightarrow\left(\text { Tot }_{1}\right)_{*-1}$ are defined by

$$
d_{j}(c, \tilde{c})=\left(d_{j} c, d_{j} \tilde{c}\right)=\left(d_{j} c, \tilde{c} \circ\left(d^{j} \times 1\right)\right),
$$

where $d^{j}: \Delta^{*-1} \rightarrow \Delta^{*}$ is the $j$-th coface. Recall that for

$$
\begin{gathered}
(a, b) \in \Delta^{t-1} \times \Delta^{1}, d^{j} \in \Delta_{t-1}^{t}, 0 \leqslant j \leqslant t, \\
\left(d_{j} \tilde{c}\right)(a, b)=\tilde{c}\left(d^{j} a, b\right) .
\end{gathered}
$$

One must be careful to distinguish between $d_{j} \tilde{c}$ and the composite $d_{j} \circ \tilde{c}$; by definition, if $(x, y) \in \Delta^{t} \times \Delta^{1}, d^{j} \in \Delta_{t-1}^{t}$, then $d_{j}(\tilde{c}(x, y))=\tilde{c}\left(x d^{j}, y d^{j}\right)$.

Writing out the conditions for $(c, \tilde{c})$ to be an element of $\left(\overline{T o t}_{1}\right)_{t}$ explicitly, in addition to the conditions above for $(c, \tilde{c}) \in\left(\operatorname{Tot}_{1}\right)_{t}$, we must also have, for every $(a, b) \in \Delta^{t} \times \Delta^{1}, \tilde{c}\left(d^{j} a, b\right)=1$ for $0 \leqslant j \leqslant t-1$, and $d_{j} c=1$, for $0 \leqslant j \leqslant t-1$.

Define a nonabelian chain complex $G(C)$ as follows.

$$
G(C)_{0}=\left\{(z, w) \in C_{0}^{0} \times C_{1}^{1} \mid d^{0} z=d_{0} w, d^{1} z=d_{1} w, s_{0} z=s^{0} w\right\},
$$

and for $i>0$,

$$
\begin{aligned}
G(C)_{i} & =\left\{\left(c, A_{0}, \ldots, A_{i}\right) \in \overline{C_{i}^{0}} \times\left(C_{i+1}^{1}\right)^{i+1} \mid d_{0} A_{0}=d^{0} c ; d_{i+1} A_{i}=d^{1} c ;\right. \\
d_{l} A_{j} & =1,0 \leqslant l<j \leqslant i \text { or } j+1<l \leqslant i ; \\
d_{j+1} A_{j} & \left.=d_{j+1} A_{j+1}, 0 \leqslant j<i ; s^{0} A_{j}=s_{j} c, 0 \leqslant j \leqslant i\right\} .
\end{aligned}
$$


The differentials are, for $i \geqslant 1$,

$$
\begin{gathered}
d: G(C)_{i} \rightarrow G(C)_{i-1}, \\
d\left(c, A_{0}, \ldots, A_{i}\right)=\left(d_{i} c, d_{i+1} A_{0}, \ldots, d_{i+1} A_{i-1}\right) .
\end{gathered}
$$

Note that for every $i, G(C)_{i}$ is a subgroup of $\bar{C}_{i}^{0} \times\left(\left(C^{1}\right)^{I}\right)_{i}$, using the definition of $\left(C^{1}\right)^{I}$ from Section 2.3.1.

It is straightforward to check that $d$ is a homomorphism that really takes values in the indicated group, and that $d \circ d$ is constant at 1 .

We may envision the criteria for $\left(c, A_{0}, \ldots, A_{i}\right)$ to be an element of $G(C)_{i}$ in an $(i+2) \times(i+1)$-matrix, whose $k, l$ entry is $d_{k} A_{l}$, and whose top $i+1$ rows form a lower triangular band matrix, with entries of 1 below the first subdiagonal:

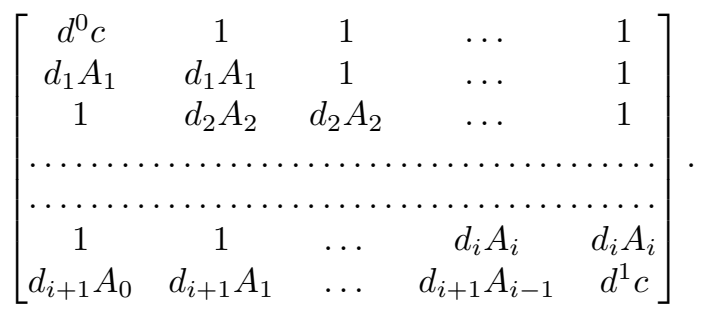

Note that the first $i$ entries of the last row form the last $i$ components of $d\left(c, A_{0}, \ldots, A_{i}\right)$.

Theorem 2.4. There is a natural isomorphism of (nonabelian) chain complexes $\tilde{\phi}: \overline{\text { Tot }_{1} C} \rightarrow G(C)$. Furthermore, the square (of maps of nonabelian chain complexes) below commutes:

$$
\begin{aligned}
& \overline{\operatorname{Tot}_{1} C} \rightarrow G(C) \\
& \frac{\downarrow}{\operatorname{Tot}_{0} C}=\frac{\downarrow}{C^{0}}
\end{aligned}
$$

where the left vertical arrow is induced by the fibration $\operatorname{Tot}_{1} C \rightarrow$ Tot $_{0} C$, and the right vertical arrow is the map $\left(c, A_{0}, \ldots\right) \mapsto c$.

Proof. The map $\tilde{\phi}$, in all degrees, is induced by the isomorphism of simplicial groups

$$
\phi: \operatorname{Hom}\left(\Delta^{1}, C^{1}\right) \rightarrow\left(C^{1}\right)^{I}
$$

of Lemma 2.3; i.e.,

$$
\tilde{\phi}(c, \tilde{c}) \doteq(c, \phi(\tilde{c})) .
$$

Since $\phi$ is an isomorphism, $\tilde{\phi}$ is injective. 
One can check:

- If $(c, \tilde{c}) \in{\overline{\operatorname{Tot}}{ }_{1} C_{j}}_{\subseteq} \bar{C}_{j}^{0} \times{\overline{\operatorname{Hom}\left(\Delta^{1}, C^{1}\right)}}_{j}$, then

$$
(c, \phi(\tilde{c})) \in G(C)_{j} \subseteq \bar{C}_{j}^{0} \times \overline{\left(C^{1}\right)_{j}^{I}} .
$$

- $\tilde{\phi}$ is a map of chain complexes.

Finally, we do check that $\tilde{\phi}$ is surjective, leaving the degree zero case to the reader.

If $\left(c, A_{0}, \ldots, A_{j}\right) \in G(C)_{j}$, then there is a unique $\tilde{c} \in \operatorname{Hom}\left(\Delta^{1}, C^{1}\right)_{j}$ such that $\phi(\tilde{c})=\left(A_{0}, \ldots, A_{j}\right) \in\left(C_{j+1}^{1}\right)^{j+1}$, and we only need to show that $(c, \tilde{c}) \in \operatorname{Tot}_{1} C$.

Since $\left(c, A_{0}, \ldots, A_{j}\right) \in G(C)$,

$$
d^{0} c=d_{0} A_{0}=d_{0}\left(\tilde{c}\left(s^{0}, s^{1} \cdots s^{j}\right)\right) \doteq \tilde{c}\left(s^{0} d^{0}, s^{1} \cdots s^{j} d^{0}\right)=\tilde{c}\left(i d, d^{0} s^{0} \cdots s^{j-1}\right),
$$

and

$$
d^{1} c=d_{j+1} A_{j}=d_{j+1}\left(\tilde{c}\left(s^{j}, s^{0} \cdots s^{j-1}\right)\right) \doteq \tilde{c}\left(i d, d^{1} s^{0} \cdots s^{j-1}\right) .
$$

Now, if $a \in \Delta^{j}$, then, abusing notation, and letting $d^{0}, d^{1} \in \Delta^{1}$ be the constant maps at 1,0 respectively (in any simplicial degree), since $d^{0}=d^{0} a$, and $d^{1}=d^{1} a$, we must have

$$
\tilde{c}\left(a, d^{\epsilon}\right)=\tilde{c}\left(a, d^{\epsilon} a\right)=a^{*}\left(\tilde{c}\left(i d, d^{\epsilon}\right)\right)=a^{*} d^{\epsilon} c .
$$

Also, for $0 \leqslant k \leqslant j, s_{k} c=s^{0} A_{k}=s^{0}\left(\tilde{c}\left(s^{k}, s^{0} \cdots \hat{s}^{k} \cdots s^{j}\right)\right)$.

Thus,

$$
\begin{gathered}
c=d_{j} s_{j} c=s^{0}\left(\tilde{c}\left(s^{j} d^{j}, s^{0} \cdots s^{j-1} d^{j}\right)\right)=s^{0}\left(\tilde{c}\left(i d, d^{0} s^{0} \cdots s^{j-1}\right)\right), \\
c=d_{j+1} s_{j} c=s^{0}\left(\tilde{c}\left(s^{j} d^{j+1}, s^{0} \cdots s^{j-1} d^{j+1}\right)\right)=s^{0}\left(\tilde{c}\left(i d, d^{1} s^{0} \cdots s^{j-1}\right)\right),
\end{gathered}
$$

and, if $0 \leqslant k<j$,

$$
c=d_{k} s_{k} c=s^{0}\left(\tilde{c}\left(s^{k} d^{k}, s^{0} \cdots \hat{s}^{k} \cdots s^{j} d^{k}\right)\right)=s^{0}\left(\tilde{c}\left(i d, s^{0} \cdots \hat{s}^{k-1} \cdots s^{j-1}\right)\right) .
$$

Thus, since every $b \in \Delta_{j}^{1}$ is either of the form

$$
d^{0} s^{0} \cdots s^{j-1}, d^{1} s^{0} \cdots s^{j-1} \quad \text { or } \quad s^{0} \cdots \hat{s}^{k-1} \cdots s^{j-1},
$$

one can use the equations immediately above to check that

$$
a^{*} c=s^{0}(\tilde{c}(a, b)),
$$

for every $(a, b) \in \Delta^{j} \times \Delta^{1}$.

Similar models may be defined for the nonabelian chain complexes $\overline{\operatorname{Tot}}_{q}$, for every $q$, but we do not present these here.

\subsection{Homotopy properties}

We now discuss some homotopical properties of the constructions $\mathbf{P}, \boldsymbol{\Omega}$, Tot $_{q}$ and Tot. For these closed-model-theoretic properties, we do not present details.

\section{Lemma 2.5.}

(a) The functors $\mathbf{P}$ and $\boldsymbol{\Omega}$ preserve fibrations, homotopy fibrations and weak equivalences in the category of simplicial groups, as do their extensions to the category of cosimplicial simplicial groups. 
(b) The functors Tot $t_{q}$ and Tot from the category of cosimplicial simplicial groups to that of simplicial groups preserve fibrations, homotopy fibrations and weak equivalences.

Proof. Both of these follow from the following two facts, and the definitions:

- In the appropriate closed (simplicial) model categories, $\Delta^{\epsilon}, S k_{q}(\Delta)$ and $\Delta$ are all cofibrant objects, and $G$ (whether a simplicial group or a cosimplicial simplicial group) is a fibrant object $([4, \mathrm{Ch} . \mathrm{X}, 4])$.

- Since the constructions are being performed in an appropriate closed (simplicial) model category $\mathcal{C}$, "axiom SM7" holds $([\mathbf{1 5}],[\mathbf{4}$, Ch. X], $[\mathbf{8}]$; see also $[\mathbf{1 9}$, Section $5])$; having as a corollary that the functor $\operatorname{Hom}_{\mathcal{C}}(A,-)$ preserves fibrations and weak equivalences between fibrant objects, if $A$ is a cofibrant object.

Lemma 2.6. Suppose $G$ and $H$ are cosimplicial simplicial groups, with a simplicialwise weak equivalence $\theta: G \rightarrow \boldsymbol{\Omega} H$ that is also a homomorphism of groups. Then, for every $q \geqslant 0$,

(a) $\theta$ induces a weak equivalence of simplicial groups $\theta: \operatorname{Tot}_{q} G \rightarrow \operatorname{Tot}_{q} \boldsymbol{\Omega} H$, compatible with the fibrations in the Tot-towers for $G$ and $\Omega H$.

(b) Furthermore, there is a natural isomorphism of simplicial groups

$$
\operatorname{Tot}_{q} \mathbf{P} H \rightarrow \mathbf{P} \operatorname{Tot}_{q} H
$$

inducing an isomorphism of fibrations from the fibration sequence

$$
\operatorname{Tot}_{q} \mathbf{\Omega H} \rightarrow \operatorname{Tot}_{q} \mathbf{P} H \rightarrow \operatorname{Tot}_{q} H
$$

to the fibration sequence

$$
\boldsymbol{\Omega} \operatorname{Tot}_{q} H \rightarrow \mathbf{P} \operatorname{Tot}_{q} H \rightarrow \operatorname{Tot}_{q} H .
$$

These isomorphisms are all compatible with the fibrations in the Tot-towers.

(c) If $q \geqslant 1$, and if $F_{q} G$ is the kernel of $p: \operatorname{Tot}_{q} G \rightarrow \operatorname{Tot}_{q-1} G$ while $F_{q} H$ is the kernel of $p: \operatorname{Tot}_{q} H \rightarrow \operatorname{Tot}_{q-1} H$, then $\theta$ induces a natural homomorphism of simplicial groups $F_{q} G \rightarrow \boldsymbol{\Omega} F_{q} H$ that is a weak equivalence.

(d) Thus (for every $q \geqslant 0), \pi_{j}\left(\operatorname{Tot}_{q} G\right)$ and $\pi_{j}\left(F_{q} G\right)$ are abelian groups, for every $j \geqslant 0$.

Proof. Item (b) is a consequence of "adjointness" ([8, Lemma 2.3]); there are natural isomorphisms of simplicial groups, for $\epsilon=0,1$

$$
\begin{aligned}
\operatorname{Hom}_{\mathcal{S}}\left(\Delta^{\epsilon}, \operatorname{Tot}_{q} G\right) & =\operatorname{Hom}_{\mathcal{S}}\left(\Delta^{\epsilon}, \operatorname{Hom}_{c \mathcal{S}}\left(S k_{q}(\Delta), G\right)\right) \\
& \cong \operatorname{Hom}_{c \mathcal{S}}\left(S k_{q}(\Delta), \operatorname{hom}\left(\Delta^{\epsilon}, G\right)\right)
\end{aligned}
$$

and the definitions; recall here that the cosimplicial simplicial group $\operatorname{hom}\left(\Delta^{\epsilon}, G\right)$ is defined by

$$
\operatorname{hom}\left(\Delta^{\epsilon}, G\right)^{n}=\operatorname{Hom}_{\mathcal{S}}\left(\Delta^{\epsilon}, G^{n}\right)
$$


For item (c), consider the following diagram, in which the columns are fibration sequences and in the bottom two rows, the arrows are weak equivalences:

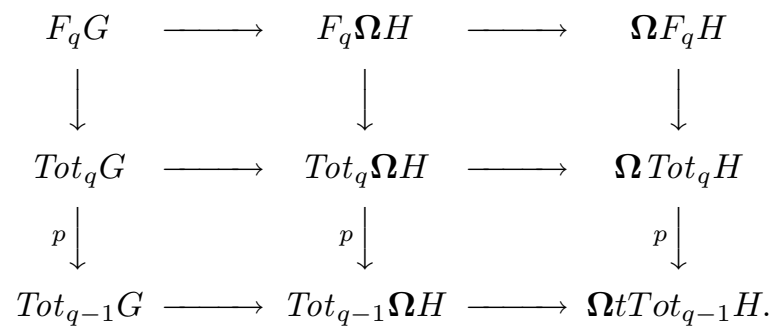

This means that the top row arrows are weak equivalences too.

\section{An extension of the spectral sequence for Tot}

In Section 3.1 we first outline the general construction of the Bousfield-Kan spectral sequence [4] for any cosimplicial simplicial group $X$. Then, in 3.2, we show how an extension of the spectral sequence may be constructed, and, in 3.3, discuss its convergence properties in detail when our cosimplicial simplicial group is cofinite and looped. In particular, as one might expect, the condition of being looped means that the spectral sequence is no longer "fringed", and the condition of being cofinite means that the spectral sequence converges strongly to the homotopy groups of $\operatorname{Tot}(X)$. The purpose of this is to give an exposition of a (known) remedy for the defects of the Bousfield-Kan spectral sequence, found not by passing to the category of spectra but, instead, by restricting to the category of cosimplicial simplicial groups, with the applications begun in this paper as motivation.

\subsection{The general construction for cosimplicial simplicial groups}

Let $X$ be a cosimplicial simplicial group.

The Bousfield-Kan spectral sequence is the homotopy spectral sequence (functorial in $X$ ) for the tower of fibrations

$$
\cdots \stackrel{p}{\rightarrow} \operatorname{Tot}_{q} X \stackrel{p}{\rightarrow} \operatorname{Tot}_{q-1} X \stackrel{p}{\rightarrow} \cdots \stackrel{p}{\rightarrow} \operatorname{Tot}_{0} X .
$$

Let $F_{q}=F_{q} X \subseteq \operatorname{Tot}_{q} X$ be the kernel of the homomorphism

$$
p: \operatorname{Tot}_{q} X \rightarrow \operatorname{Tot}_{q-1} X, \text { for every } q \geqslant 1 .
$$

If $q=0$, define $F_{0} X=\operatorname{Tot}_{0} X=X^{0}$.

We may compute the homotopy groups of simplicial groups as in Section 2, using the notation introduced there.

As in Section 2, we have (functorial in $X$ ) long exact sequences of homotopy groups (note also that every map is a group homomorphism, and that every group is abelian, except possibly the zero-th groups $\pi_{0}$ ), for $q \geqslant 1$ and $n \geqslant 0$ :

$$
\cdots \rightarrow \pi_{n+1}\left(\operatorname{Tot}_{q-1} X\right) \stackrel{\delta}{\rightarrow} \pi_{n}\left(F_{q}\right) \rightarrow \pi_{n}\left(\operatorname{Tot}_{q} X\right) \rightarrow \pi_{n}\left(\operatorname{Tot}_{q-1} X\right) \stackrel{\delta}{\rightarrow} \cdots .
$$

For $r \geqslant 0$, and $j \geqslant 0$, define

$$
\pi_{j}^{r}\left(\operatorname{Tot}_{q} X\right)=\operatorname{image}\left(p_{*}^{r}: \pi_{j}\left(\operatorname{Tot}_{q+r} X\right) \rightarrow \pi_{j}\left(\operatorname{Tot}_{q} X\right)\right) .
$$

This is always a subgroup of $\pi_{j}\left(\operatorname{Tot}_{q} X\right)$. 
Note that if $r=0$, then $\pi_{j}^{0}\left(\operatorname{Tot}_{q} X\right)=\pi_{j}\left(\operatorname{Tot}_{q} X\right)$.

For $t-s \geqslant 0, r \geqslant 1$, define

$$
Z_{r}^{s, t}=\operatorname{ker}\left(\pi_{t-s}\left(F_{s}\right) \rightarrow \pi_{t-s}\left(\operatorname{Tot}_{s} X\right) / \pi_{t-s}^{r-1}\left(\operatorname{Tot}_{s} X\right)\right) \subseteq \pi_{t-s}\left(F_{s}\right)
$$

and

$$
B_{r}^{s, t}=\delta\left(\operatorname{ker}\left(\pi_{t-s+1}\left(\operatorname{Tot}_{s-1} X\right) \stackrel{p_{*}^{r-1}}{\rightarrow} \pi_{t-s+1}\left(\operatorname{Tot}_{s-r} X\right)\right)\right) \subseteq \pi_{t-s}\left(F_{s}\right) .
$$

Note that $B_{r}^{s, t} \subseteq Z_{r}^{s, t}$ whenever defined.

Now, if $t-s \geqslant 1$ and $r \geqslant 1$, then the groups

$$
\pi_{t-s}\left(F_{s}\right), \pi_{t-s}\left(\operatorname{Tot}_{s} X\right), \pi_{t-s}^{r-1}\left(\operatorname{Tot}_{s} X\right), \pi_{t-s+1}\left(\operatorname{Tot}_{s-1} X\right), \pi_{t-s+1}\left(\operatorname{Tot}_{s-r} X\right)
$$

are all abelian groups; thus all indicated quotient groups above, as well as the quotient groups $Z_{r}^{s, t} / B_{r}^{s, t}$ are well-defined. (If $s-r<0$, we define the homotopy groups $\pi_{\alpha}\left(\operatorname{Tot}_{s-r} X\right) \doteq\{1\}$, for every $\alpha$.)

However, when $t-s=0$ and $r \geqslant 1$, and if any zero-th homotopy groups are nonabelian, we need to discuss the meaning of

$$
\pi_{0}\left(\operatorname{Tot}_{s} X\right) / \pi_{0}^{r-1}\left(\operatorname{Tot}_{s} X\right)
$$

and

$$
\operatorname{ker}\left(\pi_{0}\left(F_{s}\right) \rightarrow \pi_{0}\left(\operatorname{Tot}_{s} X\right) / \pi_{0}^{r-1}\left(\operatorname{Tot}_{s} X\right)\right) .
$$

In general, one cannot assume that $\pi_{0}^{r-1}\left(\operatorname{Tot}_{s} X\right)$ is a normal subgroup of $\pi_{0}\left(\operatorname{Tot}_{s} X\right)$. However, it is a subgroup, and thus one can form the set of cosets

$$
\pi_{0}\left(\operatorname{Tot}_{s} X\right) / \pi_{0}^{r-1}\left(\operatorname{Tot}_{s} X\right) \text {. }
$$

Let $i_{*}: \pi_{0}\left(F_{s}\right) \rightarrow \pi_{0}\left(\operatorname{Tot}_{s} X\right)$ be the homomorphism of groups induced by the inclusion of simplicial groups $F_{s} \rightarrow \operatorname{Tot}_{s} X$. The set

$$
Z_{r}^{s, s}=Z=\operatorname{ker}\left(\pi_{0}\left(F_{s}\right) \rightarrow \pi_{0}\left(\operatorname{Tot}_{s} X\right) / \pi_{0}^{r-1}\left(\operatorname{Tot}_{s} X\right)\right)
$$

is, by definition, equal to the subgroup $i_{*}^{-1}\left(\pi_{0}^{r-1}\left(\operatorname{Tot}_{s} X\right)\right)$ of $\pi_{0}\left(F_{s}\right)$. However, we know that since

$$
\operatorname{im}\left(\delta: \pi_{1}\left(\operatorname{Tot}_{s-1} X\right) \rightarrow \pi_{0}\left(F_{s}\right)\right)
$$

is a central subgroup of $\pi_{0}\left(F_{s}\right), B_{r}^{s, s}$ is a central subgroup of $Z_{r}^{s, s}$ for every $r \geqslant 1$. Thus, one can form the quotient group

$$
Z_{r}^{s, s} / B_{r}^{s, s} \doteq \pi_{0}^{r-1}\left(F_{s}\right) .
$$

Note that if all the zero-th homotopy groups are abelian, then the above definition of $E_{r}^{s, s}$ coincides with the previously given one.

The homotopy spectral sequence $\left\{E_{r}^{s, t}(X)\right\}$ is then given by:

$$
E_{r}^{s, t}(X)=Z_{r}^{s, t} / B_{r}^{s, t} \doteq \pi_{t-s}^{r-1}\left(F_{s}\right),
$$

for $t \geqslant s \geqslant 0, r \geqslant 1$ with differentials (unfortunately with the same name as face operators)

$$
d_{r}: E_{r}^{s, t} \rightarrow E_{r}^{s+r, t+r-1},
$$


defined by the composite relations

$$
\pi_{t-s}^{r-1}\left(F_{s}\right) \stackrel{i_{*}}{\rightarrow} \pi_{t-s}^{r-1}\left(\operatorname{Tot}_{s} X\right) \stackrel{\delta \circ\left(p_{*}^{r-1}\right)^{-1}}{\longrightarrow} \pi_{t-s-1}^{r-1}\left(F_{s+r}\right),
$$

for $t-s \geqslant 1$.

This spectral sequence has the following properties:

Theorem 3.1 ([4, X.6.3, X.7]; [8, VIII $])$. Let $X$ be a cosimplicial simplicial group.

(i) There are natural isomorphisms of groups ([4,X.6]) for $t-s \geqslant 0$

$$
E_{1}^{s, t} \doteq \pi_{t-s}\left(F_{s}\right) \cong \pi_{t}\left(N X^{s}\right) \cong N\left(\pi_{t}\left(X^{s}\right)\right)
$$

Note that the above groups are abelian except possibly when $t-s=0$.

(ii) There are natural isomorphisms of groups ([8, VIII.1]) for $t-s \geqslant 0$

$$
E_{2}^{s, t} \cong \pi^{s}\left(\pi_{t}(X)\right)
$$

Note that the above groups are abelian except possibly when $t=s=0$.

(iii) For $t-s>0$ and for $r \geqslant 1$, the differential $d_{r}: E_{r}^{s, t} \rightarrow E_{r}^{s+r, t+r-1}$ is a group homomorphism whose domain is always abelian. For $t-s>0$, the group $E_{r}^{s, t} \cap \operatorname{im}\left(d_{r}\right)$ is an abelian subgroup of the abelian group $E_{r}^{s, t} \cap \operatorname{ker}\left(d_{r}\right)$ and $E_{r+1}^{s, t}$ is naturally isomorphic to the quotient group

$$
\left(E_{r}^{s, t} \cap \operatorname{ker}\left(d_{r}\right)\right) /\left(E_{r}^{s, t} \cap \operatorname{im}\left(d_{r}\right)\right) .
$$

(iv) If $r \geqslant 1$, then the relation $d_{r}: E_{r}^{s-r, s-r+1} \rightarrow E_{r}^{s, s}$ is a homomorphism of groups (the domain group is always abelian), $\operatorname{im}\left(d_{r}\right)$ is a central subgroup of $E_{r}^{s, s}$, and $E_{r+1}^{s, s}$ is isomorphic to a subgroup of the quotient group $E_{r}^{s, s} /\left(\operatorname{im}\left(d_{r}\right)\right)$.

Parts (iii) and (iv) of the above theorem follow directly from the definitions and the fact that $\operatorname{im}\left(\delta: \pi_{0}\left(\operatorname{Tot}_{s-1} X\right) \rightarrow \pi_{0}\left(F_{s}\right)\right)$ is always a central subgroup of $\pi_{0}\left(F_{s}\right)$; the more difficult identifications of the $E_{1}$ and $E_{2}$ terms (parts (i) and (ii)) are done in the cited references.

We will refer to the above spectral sequence as the Bousfield-Kan spectral sequence (abbreviated BKSS) for $X$, and use the notation $\left\{E_{r}^{s, t}(X) ; d_{r}\right\}$ for this spectral sequence.

\subsection{The extended spectral sequence}

Now, let $(G, \theta, H)$ be a looped cosimplicial simplicial group. As we have seen, all the homotopy groups involved in the construction of the BKSS for $G$ are abelian; all but possibly the zero-th groups involved in the construction of the BKSS for $H$ are abelian.

Construct the BKSS's for $G, H, \mathbf{P} H$ and $\boldsymbol{\Omega} H$. Since these spectral sequences arise from the long exact sequences of homotopy groups associated to the fibrations $\operatorname{Tot}_{q} \rightarrow \operatorname{Tot}_{q-1}$, one sees using Lemma 2.6 that the construction of the entire spectral sequence associated to $G$ is isomorphic to the construction for $\Omega H$, via $\theta_{*}$. Also, using Lemmas 2.2 and 2.6, the connecting homomorphisms $\Delta$ for all the long exact sequences in homotopy obtained by applying appropriate functors $\operatorname{Tot}_{q}$ to the 
fibration $\boldsymbol{\Omega} H \rightarrow \mathbf{P} H \rightarrow H$ give isomorphisms $\pm \Delta$ of the construction of the spectral sequence for $\boldsymbol{\Omega} H$ with that of $H$. More precisely, for example, there are isomorphisms

$$
A_{r}^{s, t+1}(H) \stackrel{(-1)^{t-s+1} \Delta}{\longrightarrow} A_{r}^{s, t}(\boldsymbol{\Omega} H) \stackrel{\theta_{*}}{\longleftarrow} A_{r}^{s, t}(G),
$$

for $t-s \geqslant 0 ; s, t \geqslant 0$, and $A$ can stand for $B, Z$ or $E$. These isomorphisms commute with all the differentials where defined. We need to use $(-1)^{t-s+1} \Delta$ (the "other" boundary homomorphism in [5]) instead of $\Delta$ since by Lemma 2.2, $-\Delta \circ \delta=\delta \circ \Delta$, and the construction of $d_{r}$ involves $\delta$; indeed, the following diagram commutes:

$$
\begin{array}{rlll}
\pi_{t-s}^{r-1}\left(F_{s}(\boldsymbol{\Omega} H)\right) & \stackrel{i_{*}}{\longrightarrow} \pi_{t-s}^{r-1}\left(\operatorname{Tot}_{s} \boldsymbol{\Omega} H\right) & \stackrel{\delta \circ\left(p_{*}^{r-1}\right)^{-1}}{\longrightarrow} \pi_{t-s-1}^{r-1}\left(F_{s+1} \boldsymbol{\Omega} H\right) \\
(-1)^{t-s+1} \Delta \uparrow & (-1)^{t-s+1} \Delta \uparrow & (-1)^{t-s} \Delta \uparrow \\
\pi_{t-s+1}^{r-1}\left(F_{s} H\right) & \stackrel{i_{*}}{\longrightarrow} \pi_{t-s+1}^{r-1}\left(\operatorname{Tot}_{s} H\right) & \stackrel{\delta \circ\left(p_{*}^{r-1}\right)^{-1}}{\longrightarrow} \pi_{t-s}^{r-1}\left(F_{s+1} H\right) .
\end{array}
$$

We define one more set of "differentials"

$$
\tilde{d}_{r}(G): E_{r}^{s, s}(G) \rightarrow Y_{r}^{s+r, s+r-1}(G)
$$

for every $s \geqslant 0$ and $r \geqslant 1$, as follows.

The receiving groups $Y_{r}^{s+r, s+r-1}(G)$ are defined by $Y_{r}^{s+r, s+r-1}(G)=E_{r}^{s+r, s+r}(H)$ (note that this is always a group, but not necessarily an abelian group), and define $\tilde{d}_{r}(G)$ by $\tilde{d}_{r}(G)=d_{r}(H) \circ \Delta^{-1} \circ \theta_{*}$ :

$$
E_{r}^{s, s}(G) \stackrel{\theta_{*}}{\longrightarrow} E_{r}^{s, s}(\boldsymbol{\Omega} H) \stackrel{\Delta}{\longleftarrow} E_{r}^{s, s+1}(H) \stackrel{d_{r}(H)}{\longrightarrow} E_{r}^{s+r, s+r}(H) .
$$

The homomorphisms $\tilde{d}_{r}(G)$ also depend on the weak equivalence $\theta: G \rightarrow \boldsymbol{\Omega} H$, but we suppress this.

Now, we may augment the conclusion of part (iv) of the Theorem 3.1 by

Lemma 3.2. Let $(G, \theta, H)$ be a looped cosimplicial simplicial group. If $r \geqslant 1$, the relation

$$
d_{r}(G): E_{r}^{s-r, s-r+1}(G) \rightarrow E_{r}^{s, s}(G)
$$

is a homomorphism of abelian groups and $E_{r+1}^{s, s}$ is isomorphic to the subgroup $\operatorname{ker}\left(\tilde{d}_{r}(G)\right) /\left(\operatorname{im}\left(d_{r}(G)\right)\right)$ of the quotient group $E_{r}^{s, s}(G) /\left(\operatorname{im}\left(d_{r}(G)\right)\right)$.

The proof follows from the definitions. However, we give some details as follows.

Let $s \geqslant 0$; the homomorphism

$$
Z_{r+1}^{s, s}(G) \rightarrow E_{r}^{s, s} /\left(\operatorname{im}\left(d_{r}(G)\right)\right.
$$

is induced by the inclusion $Z_{r+1}^{s, s}(G) \subseteq Z_{r}^{s, s}(G)$. One can check, using the definitions, that the kernel of this homomorphism is precisely $B_{r+1}^{s, s}(G)$. This defines the inclusion

$$
E_{r+1}^{s, s}(G) \hookrightarrow E_{r}^{s, s} /\left(\operatorname{im}\left(d_{r}\right)\right) .
$$

(This is no different than the verification of the same result for $s \neq t$.)

Now, $z \in Z_{r+1}^{s, s}(G)$ if and only if $\Delta^{-1}\left(\theta_{*}(z)\right) \in Z_{r+1}^{s, s+1}(H)$, so we know that the class of $\Delta^{-1}\left(\theta_{*}(z)\right)$ in $E_{r}^{s, s+1}(H)$ is in $\operatorname{ker} d_{r}(H)$. 
One has the following commutative diagram of groups:

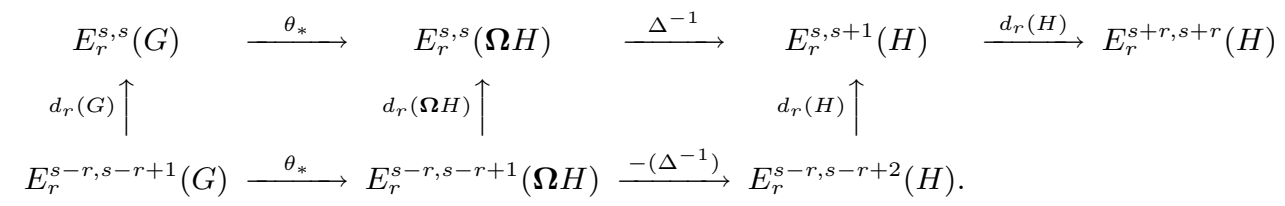

The homomorphism $\tilde{d}_{r}(G)$ is by definition the composite of the top row of arrows.

By Theorem 3.1, we know that $\operatorname{ker} d_{r}(H) / \operatorname{im}\left(d_{r}(H)\right) \cong E_{r}^{s, s+1}(H)$. The diagram above implies that the isomorphism $\operatorname{ker} d_{r}(H) \rightarrow \operatorname{ker}\left(d_{r}(H) a\right)$ induced by $a^{-1} \doteq \theta_{*}^{-1} \Delta$ gives an isomorphism $\operatorname{ker} d_{r}(H) / \operatorname{im}\left(d_{r}(H)\right) \cong \operatorname{ker}\left(d_{r}(H) a\right) / \operatorname{im}\left(d_{r}(G)\right)$.

Note that $\tilde{d}_{r}$ is natural on the category of looped cosimplicial simplicial groups.

\subsection{Convergence properties}

We now discuss the convergence of the (extended) spectral sequence $\left\{E_{r}^{s, t} ; d_{r}, \tilde{d}_{r}\right\}$, under the cofiniteness hypothesis. As one might expect, the convergence discussion is considerably simplified, but it is also extended to degree 0 . We present the details in the following. We use the notation of [4].

If $\cdots \rightarrow Z_{s} \rightarrow Z_{s-1} \rightarrow \cdots \rightarrow Z_{0} \rightarrow 1=Z_{-1}$ is any tower of fibrations of simplicial groups, then we make the following definitions, for $s, j \geqslant 0$. Here, $Z=\varliminf_{s} Z_{s}$. All of the sets defined are groups (all abelian, unless $j=0$ ) and all of the maps are group homomorphisms.

Define

$$
Q_{s} \pi_{j}(Z)=\operatorname{im}\left(\pi_{j}(Z) \rightarrow \pi_{j}\left(Z_{s}\right)\right)
$$

for $s \geqslant-1, j \geqslant 0$.

Note that $Q_{s} \pi_{j}(Z) \rightarrow Q_{s-1} \pi_{j}(Z)$ is a surjection as indicated, for $j \geqslant 0, s \geqslant 0$.

$$
e_{\infty}^{s, s+j} \doteq \operatorname{ker}\left(Q_{s} \pi_{j}(Z) \rightarrow Q_{s-1} \pi_{j}(Z)\right),
$$

for $s, j \geqslant 0$.

$$
E_{\infty}^{s, s+j} \doteq \cap_{r>s} E_{r}^{s, s+j}
$$

for $s, j \geqslant 0$.

Now, if $F_{s, j}=F_{s, j}(Z)=\operatorname{ker}\left(\pi_{j}(Z) \rightarrow \pi_{j}\left(Z_{s}\right)\right)$, for $s, j \geqslant 0$, then

$$
Q_{s} \pi_{j}(Z) \cong \pi_{j}(Z) / F_{s, j}
$$

for every $s, j \geqslant 0$, and there exists a filtration

$$
\cdots \subseteq F_{s+1, j} \subseteq F_{s, j} \subseteq \cdots \subseteq F_{0, j} \subseteq \pi_{j}(Z)=F_{-1, j}
$$

of $\pi_{j}(Z)$ by normal subgroups, for every $j \geqslant 0$. Also,

$$
e_{\infty}^{s, s+j} \cong F_{s-1, j} / F_{s, j}
$$

for every $s, j \geqslant 0$.

We see directly from the definitions that $Q_{s} \pi_{j}(Z)$ is a subgroup of $\pi_{j}^{r}\left(Z_{s}\right)$, for every $r \geqslant 1$ and $s, j \geqslant 0$ and that there are natural inclusions of groups

$$
e_{\infty}^{s, s+j} \hookrightarrow E_{\infty}^{s, s+j}
$$

for every $s, j \geqslant 0$. 
We will assume that $Z=\operatorname{Tot}(X), Z_{s}=\operatorname{Tot}_{s} X$ for some cosimplicial simplicial group $X$ and that the tower of fibrations is the Tot-tower.

Now, if $X$ is any cosimplicial simplicial group, then the fibre $F_{s+1} X$ of

$$
\operatorname{Tot}_{s+1} X \stackrel{p}{\rightarrow} \operatorname{Tot}_{s} X
$$

has homotopy groups

$$
\pi_{j}\left(F_{s+1} X\right) \cong \pi_{j+s+1}\left(N X^{s+1}\right) \cong N \pi_{j+s+1}\left(X^{s+1}\right),
$$

for $j \geqslant 0$. The last isomorphism $\pi_{j+s+1}\left(N X^{s+1}\right) \cong N \pi_{j+s+1}\left(X^{s+1}\right)$ is part of Theorem 3.1, proved in [8, VIII, Lemma 1.8], and is a natural isomorphism, commuting up to sign with connecting homomorphisms in long exact sequences corresponding to fibration sequences.

3.3.1. Convergence for cofinite looped groups

Let $(G, \theta, H)$ be a cofinite looped group. The weak equivalence $\theta$ induces

$$
\theta: N G^{s} \rightarrow N \Omega H^{s}, \quad \text { for every } s .
$$

Now, if $t \geqslant s$, we know that

$$
\pi_{t-s}\left(F_{s} G\right) \cong \pi_{t-s}\left(F_{s} \Omega H^{s}\right) \cong \pi_{t-s+1}\left(F_{s} H\right),
$$

so

$$
\pi_{t}\left(N G^{s}\right) \cong \pi_{t}\left(N \Omega H^{s}\right) \cong \pi_{t+1}\left(N H^{s}\right),
$$

for $t \geqslant s$. Also, $\pi_{0}\left(F_{s+1} H\right) \cong \pi_{s+1}\left(N H^{s+1}\right)$.

Thus, if $M \geqslant 0$ is such that $\pi_{a}\left(N H^{b}\right)=1$, for every $a \geqslant 0, b \geqslant M$, then for every $j \geqslant 0, s+1 \geqslant M$,

$$
1=\pi_{j+s+2}\left(N H^{s+1}\right) \cong \pi_{j+s+1}\left(N G^{s+1}\right) \cong \pi_{j}\left(F_{s+1} G\right),
$$

and, $\pi_{0}\left(F_{s+1} H\right) \cong \pi_{s+1}\left(N H^{s+1}\right)=1$.

So,

- For every $j \geqslant 1, s+1 \geqslant M$, the fibration $p: \operatorname{Tot}_{s+1} G \rightarrow \operatorname{Tot}_{s} G$ induces an isomorphism on the $j$-th homotopy groups.

This extends to $j=0$ as well. For every $s \geqslant 0$, there is a diagram of groups and group homomorphisms, where the squares commute up to sign, the rows are exact and the vertical arrows are isomorphisms:

$$
\begin{array}{ccccccc}
\pi_{0}\left(F_{s+1} G\right) & \rightarrow & \pi_{0}\left(\operatorname{Tot}_{s+1} G\right) & \stackrel{A}{\rightarrow} & \pi_{0}\left(\operatorname{Tot}_{s} G\right) & & \\
\downarrow & & \downarrow & & & \downarrow & \\
\pi_{1}\left(F_{s+1} H\right) & \rightarrow & \pi_{1}\left(\operatorname{Tot}_{s+1} H\right) & \stackrel{B}{\rightarrow} & \pi_{1}\left(\operatorname{Tot}_{s} H\right) & \stackrel{C}{\rightarrow} & \pi_{0}\left(F_{s+1} H\right) .
\end{array}
$$

Now, for $s+1 \geqslant M, j=0$ or 1 , and $X=G$ or $H, \pi_{j}\left(F_{s+1} X\right)=1$. Therefore, $B$ and hence $A$ are isomorphisms.

So, we have shown

Lemma 3.3. For every $j \geqslant 0, s+1 \geqslant M$, the fibration $p: \operatorname{Tot}_{s+1}(G) \rightarrow \operatorname{Tot}_{s}(G)$ induces an isomorphism on the $j$-th homotopy groups.

This naturally implies the following two lemmas. 
Lemma 3.4. For every $s \geqslant M$, the fibration $\tilde{p}: \operatorname{Tot}(G) \rightarrow \operatorname{Tot}_{s} G$ is a weak equivalence in the category of simplicial groups.

Proof. Theorem 3.1, Ch. IX of [4] says that there is an exact sequence of groups, for every $j \geqslant 0$ :

$$
1 \rightarrow \lim _{s}^{1} \pi_{j+1}\left(\operatorname{Tot}_{s} G\right) \rightarrow \pi_{j}(\operatorname{Tot}(G)) \rightarrow \lim _{s} \pi_{j}\left(\operatorname{Tot}_{s} G\right) \rightarrow 1 .
$$

Since $\pi_{k}\left(\operatorname{Tot}_{s+1} G\right) \rightarrow \pi_{k}\left(\operatorname{Tot}_{s} G\right)$ is an isomorphism, for every $k \geqslant-1$ and $s+1 \geqslant M$,

$$
\lim _{s}^{1} \pi_{j+1}\left(\operatorname{Tot}_{s} G\right)=1
$$

and

$$
\lim _{s} \pi_{j}\left(\operatorname{Tot}_{s} G\right) \cong \pi_{j}\left(\operatorname{Tot}_{t} G\right)
$$

for all $t \geqslant M$. Thus,

$$
\pi_{j}(\operatorname{Tot}(G)) \cong \lim _{s} \pi_{j}\left(\operatorname{Tot}_{s} G\right) \cong \pi_{j}\left(\operatorname{Tot}_{t} G\right),
$$

for all $j \geqslant 0, t \geqslant M$.

Lemma 3.5. For every $s \geqslant 0, j \geqslant 0$ and $r \geqslant M+1, E_{\infty}^{s, s+j} \cong E_{r}^{s, s+j}$.

Proof. For $j \geqslant 1$, this is standard, since the groups $E_{r}^{s, s+j}$ are nonidentity only in the band $0 \leqslant s \leqslant M$, and for $r$ large enough, the differentials $d_{r}$ both come from and go to the identity groups outside this band. Also, one can compute $E_{r+1}^{s, s+j}$ as $\operatorname{ker} d_{r} / \operatorname{im} d_{r}$, for $j \geqslant 1$.

For $j=0$, the only problem with the same argument in this particular spectral sequence would have been the "fringing" effect: namely, that there are no outgoing differentials $d_{r}$ from the groups $E_{r}^{s, s}$, making it impossible to compute $E_{r+1}^{s, t}$ as $\operatorname{ker} d_{r} / \operatorname{im} d_{r}$. However, we have extended the spectral sequence with the differentials $\tilde{d}_{r}$; the receiving groups for these differentials are the groups

$$
Y_{r}^{s+r, s+r-1} \doteq E_{r}^{s+r, s+r}(H) .
$$

Since these have been identified as subquotients of $\pi_{0}\left(F_{s+r} H\right)$, and $s+r \geqslant M$, these groups are all identity groups, using the cofiniteness hypothesis, and the identification of $\pi_{0}\left(F_{s+r}(H)\right)$ with $\pi_{s+r}\left(N H^{s+r}\right)$.

Moreover, since we have identified $E_{r+1}^{s, s}$ as the quotient ker $\tilde{d}_{r} / \operatorname{im} d_{r}$, one may use the same argument as for $j \geqslant 1$ to make the conclusion of the lemma.

Lemma 3.6. For every $s, j \geqslant 0, e_{\infty}^{s, s+j} \cong E_{\infty}^{s, s+j}$.

Proof. Fix $s, j \geqslant 0$; let $M$ be as always. Choose $t$ so that $t$ is greater than or equal to both $s$ and $M$. Now,

$$
\begin{aligned}
Q_{s} \pi_{j}(\operatorname{Tot}(G)) & =\operatorname{im}\left(\pi_{j}(\operatorname{Tot}(G)) \rightarrow \pi_{j}\left(\operatorname{Tot}_{s} G\right)\right) \\
& =\operatorname{im}\left(\pi_{j}(\operatorname{Tot}(G)) \rightarrow \pi_{j}\left(\operatorname{Tot}_{s+t} G\right) \rightarrow \pi_{j}\left(\operatorname{Tot}_{s} G\right)\right) \\
& =\operatorname{im}\left(\pi_{j}\left(\operatorname{Tot}_{s+t} G\right) \rightarrow \pi_{j}\left(\operatorname{Tot}_{s} G\right)\right)
\end{aligned}
$$

since $s+t \geqslant M$. But

$$
\operatorname{im}\left(\pi_{j}\left(\operatorname{Tot}_{s+t} G\right) \rightarrow \pi_{j}\left(\operatorname{Tot}_{s} G\right)\right)=\pi_{j}^{t}\left(\operatorname{Tot}_{s} G\right)
$$


by definition. Similarly,

$$
Q_{s-1} \pi_{j}(\operatorname{Tot}(G)) \cong \pi_{j}^{t+1}\left(\operatorname{Tot}_{s-1} G\right) .
$$

So, there is a commutative diagram of short exact sequences:

$$
\begin{aligned}
& 1 \quad \rightarrow \quad E_{t+1}^{s, s+j}=E_{\infty}^{s, s+j} \quad \rightarrow \quad \pi_{j}^{t}\left(\operatorname{Tot}_{s} G\right) \quad \rightarrow \quad \pi_{j}^{t+1}\left(\operatorname{Tot}_{s-1} G\right) \quad \rightarrow \quad 1 \\
& 1 \rightarrow e_{\infty}^{\uparrow, s+j} \rightarrow Q_{s} \pi_{j}(\operatorname{Tot}(G)) \rightarrow Q_{s-1} \pi_{j}^{\uparrow}(\operatorname{Tot}(G)) \rightarrow 1 .
\end{aligned}
$$

$E_{t+1}=E_{\infty}$ since $t \geqslant M$; the top row is exact since $t \geqslant s$ and the rightmost two vertical arrows are isomorphisms using the previous paragraph. Thus, the left vertical arrow is an isomorphism.

In summary, we have the following:

Let $(G, \theta, H)$ be a cofinite looped cosimplicial simplicial group; the integer $M$ is chosen so that $\pi_{j}\left(N H^{q}\right)$ is the identity group, for $j \geqslant 0$ and $q>M$. Then, there is an extended Bousfield-Kan spectral sequence $\left\{E_{r}^{s, s+j} ; s, j \geqslant 0 ; d_{r}, \tilde{d}_{r}\right\}$ and a finite filtration

$$
\begin{gathered}
1=F_{M, j} \subseteq \cdots \subseteq F_{s, j} \subseteq F_{s-1, j} \subseteq \cdots \subseteq F_{0, j} \subseteq \pi_{j}(\operatorname{Tot}(G))=F_{-1, j} \\
F_{s, j}=\operatorname{ker} p_{s, j}, p_{s, j}: \pi_{j}(\operatorname{Tot}(G)) \rightarrow \pi_{j}\left(\operatorname{Tot}_{s} G\right) \\
F_{s-1, j} / F_{s, j} \cong E_{\infty}^{s, s+j}=E_{M+1}^{s, s+j}
\end{gathered}
$$

for every $s, j \geqslant 0$, and

$$
E_{2}^{s, s+j} \cong \pi^{s} \pi_{s+j}(G)
$$

for every $s, j \geqslant 0$.

One of the main points (the only point?) in being fussy about the extension of the spectral sequence is to retain information for zero-th homotopy groups.

\section{Cosimplicial replacements and the BKSS}

\subsection{Presheaves on a small category}

We review the definitions, using the notation of [7].

Let $\mathcal{I}$ be a small category. Rather than saying " $x$ is an object of $\mathcal{I}$ " we will say " $x \in \mathcal{I}$ ".

Suppose that $\mathfrak{F}$ is a functor from $\mathcal{I}$ to some category $\mathcal{C}$; for each object $x \in \mathcal{I}$ we have an object $\mathfrak{F}(x)$ of $\mathcal{C}$ and for each morphism $x \stackrel{g}{\rightarrow} y$ in $\mathcal{I}$ we have a morphism in $\mathcal{C}, \mathfrak{F}(x \stackrel{g}{\rightarrow} y): \mathfrak{F}(x) \rightarrow \mathfrak{F}(y)$, satisfying the properties necessary to be a functor.

Such a functor $\mathfrak{F}$ is called a presheaf (with values in $\mathcal{C}$ ) on the category $\mathcal{I}$, or, also, a diagram of objects in $\mathcal{C}$.

If $\mathcal{I}$ is a small category, let $\mathcal{N} \mathcal{I}$ be the nerve of $\mathcal{I}$, a simplicial set. Thus, $\mathcal{N I}_{0}$ is the set of objects of $\mathcal{I}$, and for $q \geqslant 1, \mathcal{N I}_{q}$ is the set of sequences $\left[x_{0} \stackrel{g_{1}}{\rightarrow} x_{1} \cdots \stackrel{g_{q}}{\rightarrow} x_{q}\right]$ of objects $x_{i}$ and composable morphisms $g_{i}$ as indicated. The face and degeneracy maps are defined as is usual.

In the rest of this section, the presheaf $\mathfrak{F}$ has values in the category of groups or the category of simplicial groups (not necessarily abelian). 
We give some details of the constructions we will use when the range category is that of simplicial groups. The constructions are of course formally the same for the category of groups.

Let $q \geqslant 0, j \geqslant 0$. The set of all functions

$$
f: \mathcal{N I}_{q} \rightarrow \bigcup_{x \in \mathcal{I}} \mathfrak{F}(x)_{j}
$$

such that

$$
f\left(\left[x_{0} \stackrel{g_{1}}{\rightarrow} x_{1} \rightarrow \cdots \stackrel{g_{q}}{\rightarrow} x_{q}\right]\right) \in \mathfrak{F}\left(x_{q}\right)_{j}
$$

for every $q$-simplex $\left[x_{0} \stackrel{g_{1}}{\rightarrow} x_{1} \cdots \stackrel{g_{q}}{\rightarrow} x_{q}\right]$ in $\mathcal{N} \mathcal{I}_{q}$, will be denoted by $C^{q}\left(\mathcal{I}, \mathfrak{F}_{j}\right)$.

Fixing $q$, the indexed collection

$$
\left\{C^{q}\left(\mathcal{I}, \mathfrak{F}_{j}\right) \mid j \geqslant 0\right\}
$$

forms a simplicial set: if $\theta: \mathbf{j}_{1} \rightarrow \mathbf{j}_{2}$ is an element of $\Delta_{j_{1}}^{j_{2}}$, then

$$
\theta^{*}: C^{q}\left(\mathcal{I}, \mathfrak{F}_{j_{2}}\right) \rightarrow C^{q}\left(\mathcal{I}, \mathfrak{F}_{j_{1}}\right)
$$

is defined by

$$
\theta^{*}(f)\left(\left[x_{0} \stackrel{g_{1}}{\rightarrow} x_{1} \rightarrow \cdots \stackrel{g_{q}}{\rightarrow} x_{q}\right]\right)=\theta^{*}\left(f\left(\left[x_{0} \stackrel{g_{1}}{\rightarrow} x_{1} \rightarrow \cdots \stackrel{g_{q}}{\rightarrow} x_{q}\right]\right)\right) .
$$

Notice that this simplicial set is a simplicial group under pointwise multiplication of functions.

If $\mu: \mathfrak{F} \rightarrow \mathfrak{G}$ is a natural transformation of functors from $\mathcal{I}$ to the category of simplicial groups (we call such a $\mu$ a homomorphism of presheaves of simplicial groups), then for every $q, \mu$ defines a homomorphism of simplicial groups $\mu_{\sharp}: C^{q}(\mathcal{I}, \mathfrak{F}) \rightarrow C^{q}(\mathcal{I}, \mathfrak{G})$ by the rule

$$
\mu_{\sharp}(f)\left(\left[x_{0} \stackrel{g_{1}}{\rightarrow} x_{1} \rightarrow \cdots \stackrel{g_{q}}{\rightarrow} x_{q}\right]\right)=\mu\left(x_{q}\right)\left(f\left(\left[x_{0} \stackrel{g_{1}}{\rightarrow} x_{1} \rightarrow \cdots \stackrel{g_{q}}{\rightarrow} x_{q}\right]\right) .\right.
$$

One can check that this definition of $\mu_{\sharp}$ is functorial.

For every $q \geqslant 0$, there are $q+2$ homomorphisms

$$
d^{i}: C^{q}(\mathcal{I}, \mathfrak{F}) \rightarrow C^{q+1}(\mathcal{I}, \mathfrak{F}), \quad 0 \leqslant i \leqslant q+1,
$$

where $d^{i}(f)\left(\left[x_{0} \stackrel{g_{1}}{\longrightarrow} x_{1} \rightarrow \cdots \stackrel{g_{q+1}}{\longrightarrow} x_{q+1}\right]\right)$ is defined to be

$$
\begin{cases}\left.f\left(x_{1} \stackrel{g_{2}}{\longrightarrow} \cdots \stackrel{g_{q+1}}{\longrightarrow} x_{q+1}\right]\right), & i=0 \\ f\left(\left[x_{0} \stackrel{g_{1}}{\longrightarrow} \cdots \rightarrow x_{i-1} \stackrel{g_{i+1} g_{i}}{\longrightarrow} x_{i+1} \rightarrow \cdots \stackrel{g_{q+1}}{\longrightarrow} x_{q+1}\right]\right), & 1 \leqslant i<q+1 \\ \mathfrak{F}\left(x_{q} \stackrel{g_{q+1}}{\longrightarrow} x_{q+1}\right)\left(f\left(\left[x_{0} \stackrel{g_{1}}{\longrightarrow} x_{1} \rightarrow \cdots \stackrel{g_{q}}{\longrightarrow}, x_{q}\right]\right)\right), & i=q+1 .\end{cases}
$$

For every $q \geqslant 1$, there are $q$ homomorphisms

$$
s^{i}: C^{q}(\mathcal{I}, \mathfrak{F}) \rightarrow C^{q-1}(\mathcal{I}, \mathfrak{F}), \quad 0 \leqslant i \leqslant q-1,
$$

defined by

$$
\begin{aligned}
& s^{i}(f)\left(\left[x_{0} \stackrel{g_{1}}{\longrightarrow} x_{1} \rightarrow \cdots \stackrel{g_{q-1}}{\longrightarrow} x_{q-1}\right]\right) \\
& \quad=f\left(\left[x_{0} \stackrel{g_{1}}{\longrightarrow} x_{1} \rightarrow \cdots \stackrel{g_{i}}{\longrightarrow} \rightarrow x_{i} \stackrel{i d}{\longrightarrow} x_{i} \rightarrow \cdots \stackrel{g_{q-1}}{\longrightarrow} x_{q-1}\right]\right) .
\end{aligned}
$$


One can verify that these homomorphisms $d^{i}$ and $s^{i}$ satisfy the cosimplicial relations and commute with $\mu_{\sharp}$, for every natural transformation $\mu: \mathfrak{F} \rightarrow \mathfrak{G}$.

Thus, we have a cosimplicial simplicial group $C(\mathcal{I}, \mathfrak{F})$; these cosimplicial simplicial groups vary functorially with $\mathfrak{F}$. This cosimplicial group is called often the cosimplicial replacement of the diagram of simplicial groups $\mathfrak{F}[\mathbf{4}]$.

As noted above, one can similarly define a cosimplicial group $C(\mathcal{I}, \mathfrak{G})$ functorially associated to any presheaf of groups $\mathfrak{G}$ on $\mathcal{I}$. The cohomotopy groups of these cosimplicial groups are usually denoted by

$$
H^{*}(\mathcal{I}, \mathfrak{G}),
$$

whenever they are defined.

Given a presheaf $\mathfrak{F}$ of simplicial groups on the small category $\mathcal{I}$ we may form the composites $\boldsymbol{\Omega F}$ and $\mathbf{P} \mathfrak{F}$; these too are presheaves of simplicial groups on $\mathcal{I}$, and there are natural maps of presheaves

$$
\mathbf{S F} \rightarrow \mathbf{P} \mathfrak{F} \rightarrow \mathfrak{F}
$$

on $\mathcal{I}$. One can check that there are natural isomorphisms of cosimplicial simplicial groups

$$
\boldsymbol{\Omega} C(\mathcal{I}, \mathfrak{F}) \cong C(\mathcal{I}, \Omega \mathfrak{F})
$$

and

$$
\mathbf{P} C(\mathcal{I}, \mathfrak{F}) \cong C(\mathcal{I}, \mathbf{P} \mathfrak{F}),
$$

inducing a natural isomorphism of the sequence

$$
\boldsymbol{\Omega} C(\mathcal{I}, \mathfrak{F}) \rightarrow \mathbf{P} C(\mathcal{I}, \mathfrak{F}) \rightarrow C(\mathcal{I}, \mathfrak{F})
$$

of cosimplicial simplicial groups with the sequence

$$
C(\mathcal{I}, \mathbf{\Omega} \mathfrak{F}) \rightarrow C(\mathcal{I}, \mathbf{P} \mathfrak{F}) \rightarrow C(\mathcal{I}, \mathfrak{F}) .
$$

Often, in the following, we will refer to the identifications of the last few paragraphs as equalities, using the identifications implicitly.

In particular, one can make the definition of a presheaf $\mathfrak{F}$ of looped simplicial groups on $\mathcal{I}$ and then, using the definitions and identifications as above, one can form a looped cosimplicial simplicial group $C(\mathcal{I}, \mathfrak{F})$, its total tower $\left\{\operatorname{Tot}_{s}(\mathcal{I}, \mathfrak{F}) ; p\right\}$ with inverse limit $\operatorname{Tot}(\mathcal{I}, \mathfrak{F})$ and homomorphisms $\tilde{p}: \operatorname{Tot}(\mathcal{I}, \mathfrak{F}) \rightarrow \operatorname{Tot}_{s}(\mathcal{I}, \mathfrak{F})$.

Thus, assuming that $C(\mathcal{I}, \mathfrak{F})$ is cofinite and looped, we may construct the extended Bousfield-Kan spectral sequence

$$
\left\{E_{r}^{s, s+j}(\mathcal{I}, \mathfrak{F}) ; s, j \geqslant 0 ; d_{r}, \tilde{d}_{r}\right\},
$$

with convergence properties discussed in Section 3.3.

The simplicial group $\operatorname{Tot}(\mathcal{I}, \mathfrak{F})$ may be identified with the homotopy inverse limit of the diagram of simplicial groups $\mathfrak{F}$ on the small category $\mathcal{I}([4, \mathrm{XI}, 5])$, but we will continue to use the Tot notation rather than the homotopy inverse limit notation $\operatorname{holim}_{\mathcal{I}} \mathfrak{F}$. 
In any case, for a presheaf of simplicial groups $\mathfrak{F}$ on $\mathcal{I}$, one always has a natural augmentation of the cosimplicial simplicial group $C(\mathcal{I}, \mathfrak{F})$ :

$$
\nu_{0}: \lim _{\mathcal{I}} \mathfrak{F} \rightarrow C(\mathcal{I}, \mathfrak{F})
$$

where $\lim _{\mathcal{I}} \mathfrak{F}$ is the ordinary inverse limit of the diagram of simplicial groups given by $\mathfrak{F}$. Indeed, this is the maximal augmentation of the cosimplicial simplicial group $C(\mathcal{I}, \mathfrak{F})$ by definition. Thus we always have a natural homomorphism of simplicial groups

$$
\tilde{\nu}_{0}:{\underset{\lim }{\longleftarrow}}_{\mathcal{I}} \mathfrak{F} \rightarrow \operatorname{Tot}(\mathcal{I}, \mathfrak{F})
$$

compatible with all the maps $\operatorname{Tot}(\mathcal{I}, \mathfrak{F}) \rightarrow \operatorname{Tot}_{s}(\mathcal{I}, \mathfrak{F})$. This natural homomorphism is not always a weak equivalence; indeed, the above two simplicial groups may not be weakly equivalent $([\mathbf{4}, \mathrm{XI}, 4.2])$.

\subsection{The identifications $E_{2}^{i, j} \cong H^{i}\left(\mathcal{I}, \pi_{j}(\mathfrak{F})\right)$ for $i=0,1$}

4.2.1. $E_{2}^{0, j}$

For any cosimplicial simplicial group $X$, and any $j \geqslant 0$, the group $E_{2}^{0, j}$ is, by definition, computed as follows.

Since $F_{0} \doteq \operatorname{Tot}_{0} X \cong X^{0}$, we see that

$$
Z_{2}^{0, j}=\pi_{j}^{1}\left(\operatorname{Tot}_{0} X\right) \subseteq \pi_{j}\left(X^{0}\right) .
$$

Now, one can prove (see, e.g., [8]) that the subgroup $Z_{2}^{0, j}$ of $\pi_{j}\left(X^{0}\right)$ is in fact exactly

$$
\left\{[t] \in \pi_{j}\left(X^{0}\right) \mid\left[d^{0} t\right]=\left[d^{1} t\right] \in \pi_{j}\left(X^{1}\right)\right\} \doteq \pi^{0} \pi_{j} X .
$$

Since $B_{2}^{0, j}$ is the identity group by definition, we have an equality

$$
E_{2}^{0, j}=\pi^{0} \pi_{j} X
$$

In the case that $X=C(\mathcal{I}, \mathfrak{F}) \doteq C, \pi^{0} \pi_{j} X$ is denoted by $H^{0}\left(\mathcal{I}, \pi_{j} \mathfrak{F}\right)$, and is also, by definition, equal to $\lim _{\mathcal{I}} \pi_{j} \mathfrak{F}$.

The homomorphism

$$
\pi_{j}(\operatorname{Tot}(\mathcal{I}, \mathfrak{F})) \stackrel{k_{1}}{\rightarrow} E_{2}^{0, j}(\mathcal{I}, \mathfrak{F})=H^{0}\left(\mathcal{I}, \pi_{j} \mathfrak{F}\right) \subseteq C^{0}\left(\mathcal{I}, \pi_{j} \mathfrak{F}\right)=\pi_{j} \operatorname{Tot}_{0}(\mathcal{I}, \mathfrak{F})
$$

is the homomorphism induced by the natural map Tot $\rightarrow$ Tot $_{0}$.

4.2.2. $E_{2}^{1, j+1}$

By definition, $E_{1}^{1, j+1}=\pi_{j}\left(F_{1}\right)$. Identify $\overline{F_{1}}$ with $\overline{\Omega N C^{1}}$. Then, the isomorphism $\pi_{j}\left(\Omega N C^{1}\right) \rightarrow \pi_{j+1} N C^{1}$ is equal to $\Delta^{-1}$, where $\Delta$ is the connecting homomorphism of the fibration

$$
\Omega N C^{1} \rightarrow P N C^{1} \rightarrow N C^{1} .
$$

Also, this connecting homomorphism may be computed with coset representatives as in Section 2.

We will be only interested in the case $j=0$ in this paper. 
Now, using the definitions, $E_{2}^{1,1}$ is a quotient of

$$
Z_{2}^{1,1}=\operatorname{ker}\left(\pi_{0} F_{1} \rightarrow \pi_{0} \operatorname{Tot}_{1} / \pi_{0} \operatorname{Tot}_{1}^{(1)}\right),
$$

where $\pi_{0} \operatorname{Tot}_{1}^{(1)}$ is the image of the homomorphism

$$
\pi_{0}\left(\operatorname{Tot}_{2}\right) \rightarrow \pi_{0}\left(\operatorname{Tot}_{1}\right)
$$

Identifying $\pi_{0} F_{1}$ with a quotient of the group

$$
\left\{A_{0} \in C_{1}^{1} \mid d_{0} A_{0}=d_{1} A_{0}=1, s^{0} A_{0}=1\right\},
$$

using Theorem 2.4, we see that the map $E_{2}^{1,1} \rightarrow \pi^{1} \pi_{1} C=H^{1}\left(\mathcal{I}, \pi_{1} \mathfrak{F}\right)$ must be defined by taking the coset determined by $A_{0}$ to the cohomology (cohomotopy) class determined by $A_{0}$. The theory (see, e.g., precisely [8, Chap. VIII, Lemma 1.19, (2)]) guarantees that all makes sense: If $\zeta$ is the coset of $A_{0}$, and $A_{0}$ also gives an element of $Z_{2}^{1,1}$, then the lemma from [8] cited above tells us that $d^{0} \zeta d^{1} \zeta^{-1} d^{2} \zeta=1$ in the abelian group $\pi_{1} C^{2}$; thus $\zeta$ represents an element of $H^{1}\left(\mathcal{I}, \pi_{1} \mathfrak{F}\right)$.

\section{Applications to algebraic $K$-theory}

We want a specific model for algebraic $K$-theory, and the one we use in this section is that given in [6]; this model, in turn, is based on [2]. We will be considering functors from a fixed small domain category $\mathcal{I}$ into various other range categories.

\subsection{Presheaves of looped simplicial groups}

This section gives the class of examples of presheaves of looped simplicial groups that we will use to apply the work of the previous sections.

We begin with the category of "star-connected" simplicial sets from [6]: A pointed simplicial set $X$, with basepoint 0 , is called "star-connected at 0 " if and only if there exists a function $\omega: X_{0} \rightarrow X_{1}$ such that $\omega(0)=s_{0}(0)$ and, for every $z \in X_{0}$, $d_{1}(\omega(z))=0$ and $d_{0}(\omega(z))=z$. The objects in the category are thus triples $(X, 0, \omega)$ where $X, 0$ and $\omega$ are as above. Morphisms are defined as expected.

There is a functor $G$ with domain category that of the star-connected simplicial sets, and range category that of simplicial groups described in two different ways in $[6]$; we denote

$$
G(X, 0, \omega) \doteq G_{\omega}(X) .
$$

We review briefly the construction of $G$, but refer to [6] for most details. One way of constructing $G$ is to use Kan's construction for a "loop" group for the (possibly) nonreduced simplicial set $X[\mathbf{1 0}]$; the function $\omega$ serves as a way to functorially associate a maximal tree to the simplicial set $X$.

In applications, the simplicial sets we use will always be nonreduced. These simplicial sets are the basic ones for algebraic $K$-theory: the classifying spaces for Quillen's category $Q P R$, associated to a ring $R$. We review the definitions and establish a change in notation in the following sections.

\subsubsection{Definitions}

The reader is referred to the standard sources (e.g., $[\mathbf{8}, \mathbf{1 4}, \mathbf{1 7}]$ ) for details in this section. 
The ring $R$ will always be a ring with a multiplicative identity; $P R$ is the category of finitely generated projective $R$-modules. Quillen's category $Q P R$ is the category whose objects are the objects of $P R$ and whose morphisms $\tau: X \cdots \rightarrow Y$ are isomorphism classes of diagrams $X \nleftarrow Z \hookrightarrow Y$ in $P R$, where, in addition, the surjective map $X \nleftarrow Z$ and the injective map $Z \hookrightarrow Y$ are admissible maps. One diagram $X \stackrel{\alpha}{\leftarrow} Z \stackrel{\beta}{\hookrightarrow} Y$ is isomorphic to another diagram $X \stackrel{\alpha_{1}}{\longleftarrow} Z_{1} \stackrel{\beta_{1}}{\hookrightarrow} Y$ if and only if there exists an isomorphism $T: Z \rightarrow Z_{1}$ such that $T \beta_{1}=\beta$ and $T \alpha_{1}=\alpha$.

The zero module 0 is an object in $Q P R$ (we do not distinguish between different zero objects); if $X$ is an object in $Q P R$, the identity morphism $i d_{X}: X \cdots \rightarrow X$ is the morphism defined by

$$
X \stackrel{i d_{X}}{\longleftarrow} X \stackrel{i d_{X}}{\longleftrightarrow} X
$$

If $\tau_{1}=X \stackrel{\alpha_{1}}{\longleftarrow} Z_{1} \stackrel{\beta_{1}}{\longleftrightarrow} Y$ and $\tau_{2}=Y \stackrel{\alpha_{2}}{\longleftarrow} Z_{2} \stackrel{\beta_{2}}{\hookrightarrow} W$ are two morphisms in $Q P R$, which are composable in the sense "first do $\tau_{1}$ and then do $\tau_{2}$ ", the composite morphism in $Q P R$ will be denoted in the usual way by $\tau_{2} \tau_{1}$. Let us recall that the composition

$$
\tau_{2} \tau_{1}=X \stackrel{\bar{\alpha}_{1}}{\longleftarrow} Z_{1} \times_{Y} Z_{2} \stackrel{\bar{\beta}_{2}}{\longleftrightarrow} W
$$

where

$$
Z_{1} \times_{Y} Z_{2}=\left\{\left(z_{1}, z_{2}\right) \in Z_{1} \times Z_{2} \mid \beta_{1}\left(z_{1}\right)=\alpha_{2}\left(z_{2}\right)\right\}
$$

and

$$
\bar{\alpha}_{1}\left(z_{1}, z_{2}\right)=\alpha_{1}\left(z_{1}\right), \bar{\beta}_{2}\left(z_{1}, z_{2}\right)=\beta_{2}\left(z_{2}\right) .
$$

Particularly simple morphisms in $Q P R$ are the morphisms defined as follows, for any projective module $P$ :

- $q_{P}$ is equal to $0 \nleftarrow P=P$.

- $\iota_{P}$ is equal to $0 \nleftarrow 0 \hookrightarrow P$.

- If $\alpha: P \rightarrow Q$ is an isomorphism of projective modules, then there is the morphism, also called $\alpha$, which is equal to $P=P \stackrel{\alpha}{\cong} Q$.

These morphisms satisfy the relations

$$
\alpha \iota_{P}=\iota_{Q}, \alpha q_{P}=q_{Q}
$$

in $Q P R$.

The simplicial set $\mathcal{N} Q P R$ is the nerve $([8])$ of the category $Q P R$ "made small"; by this we mean that $Q P R$ is replaced by an appropriate equivalent small category. However, as is customary, we shall never make a notational distinction between $Q P R$ and its equivalent small replacement. Thus, $\mathcal{N} Q P R_{0}$ consists of the objects in $Q P R$, $\mathcal{N} Q P R_{1}$ consists of the morphisms $\tau$ in $Q P R$; in general, if $q \geqslant 2$, a $q$-simplex in $\mathcal{N} Q P R_{q}$ is written as $\tau_{1}\left|\tau_{2}\right| \cdots \mid \tau_{q}$, where $\tau_{i} \in \mathcal{N} Q P R_{1}$ for every $i$, and $\tau_{i+1} \tau_{i}$ exists in $Q P R$ for $1 \leqslant i \leqslant q-1$. The face and degeneracy operators $d_{i}, s_{i}$ in $\mathcal{N} Q P R$ are defined in the usual way; we do not give the details here. 


\subsubsection{Functoriality}

Let $R, S$ be rings with 1 , and let $f: R \rightarrow S$ be a ring homomorphism. If $X_{R}=\mathcal{N} Q P R$ and $X_{S}=\mathcal{N} Q P S$, then we let $f_{*}: X_{R} \rightarrow X_{S}$ be the map of pointed simplicial sets induced by $f$ (the basepoint for both simplicial sets is 0 ). More generally, if $v$ is any exact (basepoint preserving) functor from the category $P R$ to the category $P S$, there is an induced (basepoint preserving) simplicial set map $v_{*}: \mathcal{N} Q P R \rightarrow \mathcal{N} Q P S$.

Let $\omega:\left(X_{R}\right)_{0} \rightarrow\left(X_{R}\right)_{1}$ be the function ("independent" of $R$ )

$$
\omega(P)=q_{P} .
$$

Then, we have

$$
\omega \circ f_{*}=f_{*} \circ \omega .
$$

This means that $f_{*}$ is a map in the category of triples described above; if $G=G_{\omega}$ is the functor described in [6] (and briefly reviewed below), there is an induced homomorphism of simplicial groups

$$
G f_{*}: G_{\omega}\left(X_{R}\right) \rightarrow G_{\omega}\left(X_{S}\right) .
$$

More generally, if $v$ is any (basepoint preserving) exact functor from $P R$ to $P S$, if

$$
\omega \circ v_{*}=v_{*} \circ \omega
$$

on $\mathcal{N} Q P R$ then there is an induced homomorphism of simplicial groups

$$
G v_{*}: G_{\omega}\left(X_{R}\right) \rightarrow G_{\omega}\left(X_{S}\right) .
$$

Since $\omega$ is fixed, drop it from the notation: $G R=G_{\omega}\left(X_{R}\right)$.

At this point, we review the definition of $G R$ in order to set notation, referring to $[6]$ for details.

For each $n \geqslant 0, G R_{n}$ is defined to be the free group on the set $\mathcal{N} Q P R_{n+1}$, subject to the relations:

- $s_{n} \sigma=1$, for every $\sigma \in \mathcal{N} Q P R_{n}$.

- If $\mathcal{T}$ is the (maximal) tree in $\mathcal{N} Q P R$ generated by the morphisms $q_{P}$ in $\mathcal{N} Q P R_{1}$, for every projective $P$, then $\sigma=1$ for every $\sigma \in \mathcal{T}$.

From now on, we denote the element of $G R_{n}$ corresponding to the element $\sigma \in \mathcal{N} Q P R_{n+1}$ by $t(\sigma)$.

Note that (using bar notation), for a projective module $P$,

$$
\left(1|1| \cdots \mid q_{P}\right) \in \mathcal{N} Q P R
$$

and

$$
t\left(1|1| \cdots \mid q_{P}\right)=1 ;
$$

also,

$$
t\left(\tau_{1}\left|\tau_{2}\right| \cdots \mid 1\right)=1
$$

in $G R$, for every composable sequence of morphisms $\tau_{1}, \tau_{2}, \ldots$ in $Q P R$, using the above relations. The notation 1 refers to any appropriate identity morphism, one hopes that this is not too confusing, in view of the fact that are many identity morphisms in $Q P R$. 
The definitions of the face and degeneracy maps on generators $t(\sigma) \in G R_{n}$ are

- $d_{i} t(\sigma) \doteq t\left(d_{i} \sigma\right)$, if $0 \leqslant i<n$.

- $d_{n} t(\sigma) \doteq t\left(d_{n+1} \sigma\right)^{-1} t\left(d_{n} \sigma\right)$.

- $s_{i} t(\sigma) \doteq t\left(s_{i}(\sigma)\right)$, if $0 \leqslant i \leqslant n$.

5.1.3. Loopedness

If $R$ is a ring with 1 , we let $\Sigma R$ denote the suspension of $R$ [11]; we regard $\Sigma$ as a functor from rings with 1 to rings with 1 . One has the following theorem and its corollary $([\mathbf{6}, \mathbf{2 1}])$ :

Theorem 5.1 ([6, Theorem 6.5.1]). If $R$ is a ring with 1 , then there exists a natural weak equivalence

$$
\alpha_{R}: G R \rightarrow \Omega G \Sigma R,
$$

which is also a homomorphism of groups.

In the corollary below, recall the definition of the " $i$-boundaries" $B H_{i}$ of a simplicial group $H$ from Section 2.1.

Corollary 5.2 ([6, Corollary 7.5.2]). For every $i \geqslant 0, \pi_{i}(G R)$ is a central subgroup of of $G R_{i} / B G R_{i}$.

Our functors $\mathfrak{F}$ from $\mathcal{I}$ to the category of looped simplicial groups will be obtained as composite functors. First, start with a fixed functor $\mathfrak{R}$ from $\mathcal{I}$ to the category of rings with 1 . Then, we obtain a functor $G \Re$ from $\mathcal{I}$ to the category of looped simplicial groups defined on objects by

$$
G \mathfrak{R}(v)=\left(G \Re(v), \alpha_{\mathfrak{R}(v)}, G \Sigma \mathfrak{R}(v)\right),
$$

for every object $v$ of $\mathcal{I}$.

In fact, the above functor $G \Re$ takes values in a category of "infinite loop groups", using the above theorem. However, we will not use this fact in this paper.

\subsection{The extended BKSS for the presheaf $G \Re$ on $\mathcal{I}$}

We write down the results of Section 3 for the case of the presheaf $G \Re$ on $\mathcal{I}$. We assume that $C(\mathcal{I}, G \Re)$ is cofinite. (For example, if $\mathcal{I}$ is the category associated to a finite poset, then already $N^{s} C$ vanishes for large $s$.)

Then, there is an extended Bousfield-Kan spectral sequence

$$
\left\{E_{r}^{s, s+j}(\mathcal{I}, G \Re) ; s, j \geqslant 0 ; d_{r}, \tilde{d}_{r}\right\}
$$

and a finite filtration

$$
1=F_{M, j} \subseteq \cdots \subseteq F_{s, j} \subseteq F_{s-1, j} \subseteq \cdots \subseteq F_{0, j} \subseteq \pi_{j}(\operatorname{Tot}(\mathcal{I}, G \Re))=F_{-1, j}
$$

such that

$$
F_{s-1, j} / F_{s, j} \cong E_{\infty}^{s, s+j}=E_{M+1}^{s, s+j}
$$

for every $s, j \geqslant 0$, and

$$
E_{2}^{s, s+j} \cong \pi^{s} \pi_{s+j}(C(\mathcal{I}, G \Re)) \doteq H^{s}\left(\mathcal{I}, \pi_{s+j} G \Re\right),
$$

for every $s, j \geqslant 0$. 
Using the results of [6], for every $s, j \geqslant 0$, the values $\pi_{s+j} G \mathfrak{R}(v)$ of the presheaf $\pi_{s+j} G \Re$ of abelian groups on $\mathcal{I}$ are isomorphic to the (Quillen) $K$-groups $K_{s+j}(\mathfrak{R}(v)$ ).

\subsection{Along the $(s, s)$-line}

In this section, we assume that $C(\mathcal{I}, G \Re)$ is cofinite.

We examine in this section a small part of the $(s, s)$-line of the extended BKSS.

This spectral sequence provides a filtration of

$$
\pi_{0}(\operatorname{Tot}(\mathcal{I}, G \Re)),
$$

of whose terms we write only those in which we are interested here below:

$$
F_{2,0} \subseteq F_{1,0} \subseteq F_{0,0} \subseteq \pi_{0}(\operatorname{Tot}(\mathcal{I}, G \Re)) .
$$

The successive quotients of this filtration have been identified as

$$
\begin{gathered}
\pi_{0}(\operatorname{Tot}(\mathcal{I}, G \Re)) / F_{0,0} \cong E_{\infty}^{0,0} \subseteq E_{2}^{0,0} \cong H^{0}\left(\mathcal{I}, \mathfrak{K}_{0}\right), \\
F_{0,0} / F_{1,0} \cong E_{\infty}^{1,1} \subseteq E_{2}^{1,1} \cong H^{1}\left(\mathcal{I}, \mathfrak{K}_{1}\right)
\end{gathered}
$$

and

$$
F_{1,0} / F_{2,0} \cong E_{\infty}^{2,2} \subseteq E_{3}^{2,2} \hookrightarrow H^{2}\left(\mathcal{I}, \mathfrak{K}_{2}\right) / \operatorname{im~} \partial_{2}
$$

where

$$
\partial_{2}: E_{2}^{0,1} \cong H^{0}\left(\mathcal{I}, \mathfrak{K}_{1}\right) \rightarrow E_{2}^{2,2} \cong H^{2}\left(\mathcal{I}, \mathfrak{K}_{2}\right)
$$

is the indicated differential in the (extended) BKSS.

We will often abbreviate $\pi_{i}(G \Re)$ by $\mathfrak{K}_{i}$ as above.

Therefore, there are exact sequences

- $0 \rightarrow F_{0,0} \rightarrow \pi_{0}(\operatorname{Tot}(\mathcal{I}, G \mathfrak{R})) \stackrel{p_{*}}{\rightarrow} H^{0}\left(\mathcal{I}, \mathfrak{K}_{0}\right)$,

- $0 \rightarrow F_{1,0} \rightarrow F_{0,0} \stackrel{b_{1}}{\rightarrow} H^{1}\left(\mathcal{I}, \mathfrak{K}_{1}\right)$,

- $0 \rightarrow F_{2,0} \rightarrow F_{1,0} \stackrel{c_{2}}{\rightarrow} H^{2}\left(\mathcal{I}, \mathfrak{K}_{2}\right) / \mathrm{im} d_{2}$.

5.3.1. Connections with classical $K$-theory

If $T$ is a ring with 1 , then let $K_{0}(T), K_{1}(T)$, and $K_{2}(T)$ denote the classical $K$-groups of the ring $T$; associated presheaves will be denoted by $\mathcal{K}_{i}, i=0,1,2$.

In $[\mathbf{6}]$, for $i=0,1$, explicit natural isomorphisms

$$
\xi: K_{i}(T) \rightarrow \pi_{i}(G T)
$$

are constructed; naturality then gives natural isomorphisms of cosimplicial groups

$$
\xi: C\left(\mathcal{I}, \mathcal{K}_{i}\right) \rightarrow C\left(\mathcal{I}, \mathfrak{K}_{i}\right),
$$

and, for each $j$, of groups

$$
\xi: H^{j}\left(\mathcal{I}, \mathcal{K}_{i}\right) \rightarrow H^{j}\left(\mathcal{I}, \mathfrak{K}_{i}\right) .
$$

Now, the group $K_{0}\left(\varliminf_{\lim } \mathfrak{R}\right)$ has also a filtration on it described in [7]:

$$
\mathcal{F}_{2} \subseteq \mathcal{F}_{1} \subseteq \mathcal{F}_{0} \subseteq K_{0}\left(\overleftarrow{\mathcal{I}}_{(\lim } \mathfrak{R}\right)
$$


with exact sequences

- $0 \rightarrow \mathcal{F}_{0} \rightarrow, K_{0}\left(\varliminf_{\mathcal{I}^{\prime}} \mathfrak{R}\right) \stackrel{J}{\rightarrow} H^{0}\left(\mathcal{I}, \mathcal{K}_{0}\right)$,

- $0 \rightarrow \mathcal{F}_{1} \rightarrow \mathcal{F}_{0} \stackrel{B}{\rightarrow} H^{1}\left(\mathcal{I}, \mathcal{K}_{1}\right)$,

- $0 \rightarrow \mathcal{F}_{2} \rightarrow \mathcal{F}_{1} \stackrel{C}{\rightarrow} H^{2}\left(\mathcal{I}, \mathcal{K}_{2}\right) / \operatorname{im}\left(\delta_{2} \circ \delta_{1}\right)$,

where $J, B, C$ are homomorphisms described in [7], and $\delta_{2}, \delta_{1}$ are connecting homomorphisms arising in exact sequences associated to certain nonabelian cohomology groups. The pertinent definitions are reviewed below.

Consider the homomorphism

$$
j: K_{0}(\underbrace{\lim }_{\mathcal{I}} \mathfrak{R}) \rightarrow \pi_{0}(\operatorname{Tot}(\mathcal{I}, G \Re))
$$

described by the composite

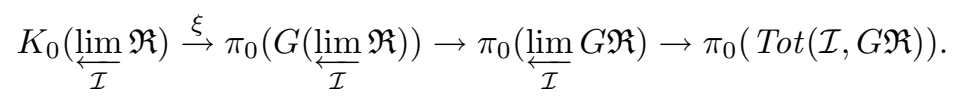

The point of this section is to prove

Theorem 5.3. The inverse image of the filtration given by the extended BKSS

$$
F_{1,0} \subseteq F_{0,0} \subseteq \pi_{0}(\operatorname{Tot}(\mathcal{I}, G \Re))
$$

under the homomorphism $j$ contains the filtration

$$
\mathcal{F}_{1} \subseteq \mathcal{F}_{0} \subseteq K_{0}(\underbrace{\lim }_{\mathcal{I}} \Re)
$$

The result $j\left(F_{2,0}\right) \subseteq \mathcal{F}_{2}$ should be true as well, and is work in progress.

5.3.2. Review of definitions and results of $[\mathbf{6}]$ and $[\mathbf{7}]$

Let $R=\lim _{\mathcal{I}} \mathfrak{R}$. For every $v \in \mathcal{I}$, let $j^{v}: R \rightarrow \mathfrak{R}(v)$ be the natural homomorphism.

We make the following abbreviations to use in following calculations. If $v \stackrel{g}{\rightarrow} w$ is an element of $\mathcal{N I}_{1}$, say $g_{*}: \mathfrak{R}(v) \rightarrow \mathfrak{R}(w)$ is the induced ring homomorphism, making $\mathfrak{R}(w)$ into an $\mathfrak{R}(v)$-module.

Define $j_{*}^{v}(P) \doteq P(v)$ and $\mathfrak{R}(v)^{n} \doteq Q(v)$, for every $v$.

If $\theta_{v}: P(v) \rightarrow Q(v)$ is an isomorphism of $\mathfrak{R}(v)$-modules for every $v$, then $g_{*} \theta_{v}$ is the isomorphism of $\mathfrak{R}(w)$-modules defined by the following diagram:

$$
\begin{array}{cccc}
g_{*}(P(v)) \doteq & P \otimes_{R} \mathfrak{R}(v) \otimes_{\mathfrak{R}(v)} \mathfrak{R}(w) & = & P(w) \\
& & \downarrow \theta_{v} \otimes 1_{\mathfrak{R}(w)} & \\
g_{*}(Q(v)) \doteq g_{*} \theta_{v} \\
(v)^{n} \otimes_{\mathfrak{R}(v)} \mathfrak{R}(w) & = & Q(w),
\end{array}
$$

where of course we know that the way $\mathfrak{R}(w)$ is regarded as an $\mathfrak{R}(v)$ module in the phrase " $\otimes_{\mathfrak{R}(v)} \mathfrak{R}(w)$ " is using the map $g_{*}$, and the equalities result from the definition of the inverse limit and standard identifications.

The homomorphism $J$ is just the natural homomorphism

$$
K_{0}(R) \rightarrow \underset{\mathcal{I}}{\lim } \mathcal{K}_{0} \doteq H^{0}\left(\mathcal{I}, \mathcal{K}_{0}\right)
$$

The subgroup $\mathcal{F}_{0}$ of $K_{0}(R)$ is defined to be the kernel of $J$. 
There are presheaves of groups $\mathcal{G L}, \mathcal{E}$ and $\mathcal{S} \mathcal{T}$ on $\mathcal{I}$ defined by the functors

$$
\begin{aligned}
& v \mapsto G L(\Re(v)), \\
& v \mapsto E(\Re(v))
\end{aligned}
$$

and

$$
v \mapsto S T(\Re(v)),
$$

where $G L(T), E(T)$ and $S T(T)$ are the general linear group, its subgroup corresponding to the elementary matrices and the Steinberg group of a ring $T$, respectively.

To define

$$
B: \mathcal{F}_{0} \rightarrow H^{1}\left(\mathcal{I}, \mathcal{K}_{1}\right)
$$

we first represent an element of ker $J \subseteq K_{0}(R)$ as $[P]-\left[R^{m}\right]$ for some finitely generated projective $R$-module $P$. Since $\mathcal{I}$ has a finite object set, without loss of generality, we may assume that for every $v \in \mathcal{I}$, there is an isomorphism $\theta_{v}: P(v) \rightarrow Q(v)$.

Then, if $v \stackrel{g}{\rightarrow} w$ is a morphism in $\mathcal{I}$, we may regard

$$
g_{*} \theta_{v} \theta_{w}^{-1}
$$

as an element of $G L(\Re(w))$. The assignment

$$
v \stackrel{g}{\rightarrow} w \mapsto g_{*} \theta_{v} \theta_{w}^{-1} \in G L(\Re(w))
$$

gives a well-defined cocycle in $C^{1}\left(\mathcal{I}, \mathcal{G} \mathcal{L}_{1}\right)$, yielding a cohomology class $\beta\left([P]-\left[R^{m}\right]\right)$ in the nonabelian cohomology set $H^{1}\left(\mathcal{I}, \mathcal{G} \mathcal{L}_{1}\right)$, which in turn yields an element of the cohomology group $H^{1}\left(\mathcal{I}, \mathcal{K}_{1}\right)$. This last cohomology class is by definition equal to $B\left([P]-\left[R^{m}\right]\right)$. The details of this construction, and the proof that $B$ is a homomorphism, are in $[7]$.

The subgroup $\mathcal{F}_{1}$ of $K_{0}(R)$ is defined to be the kernel of $B$.

Now, there are exact sequences of presheaves

$$
1 \rightarrow \mathcal{E} \stackrel{\iota}{\rightarrow} \mathcal{G L} \rightarrow \mathcal{K}_{1} \rightarrow 1
$$

and

$$
1 \rightarrow \mathcal{K}_{2} \rightarrow \mathcal{S} \mathcal{T} \rightarrow \mathcal{E} \rightarrow 1
$$

yielding long exact sequences of pointed sets in (nonabelian) cohomology

$$
\cdots \rightarrow H^{0}\left(\mathcal{I}, \mathcal{K}_{1}\right) \stackrel{\delta_{1}}{\rightarrow} H^{1}(\mathcal{I}, \mathcal{E}) \rightarrow H^{1}(\mathcal{I}, \mathcal{G} \mathcal{L}) \rightarrow \cdots
$$

and

$$
\cdots \rightarrow H^{1}(\mathcal{I}, \mathcal{S T}) \rightarrow H^{1}(\mathcal{I}, \mathcal{E}) \stackrel{\delta_{2}}{\rightarrow} H^{2}\left(\mathcal{I}, \mathcal{K}_{2}\right) .
$$

For definitions and proofs, see [7].

To define the homomorphism $C$, first define a relation $\mathbf{C} \subseteq \mathcal{F}_{1} \oplus H^{2}\left(\mathcal{I}, \mathcal{K}_{2}\right)$ by

$$
\mathbf{C}=\left\{(\phi, \nu) \mid \exists w_{\phi} \in Z^{1}(\mathcal{I}, \mathcal{E}) \text { э } \delta_{2}\left(\left\langle w_{\phi}\right\rangle\right)=\nu \text { and } \beta(\phi)=\iota_{*}\left(\left\langle w_{\phi}\right\rangle\right)\right\} ;
$$

this is an additive relation whose domain of definition is $F_{2}$ and whose indeterminacy 
is $\operatorname{im}\left(\delta_{2} \delta_{1}\right)$, and thus defines a homomorphism of groups

$$
C: \mathcal{F}_{1} \rightarrow H^{2}\left(\mathcal{I}, \mathcal{K}_{2}\right) / \operatorname{im}\left(\delta_{2} \delta_{1}\right)
$$

Again, for details see [7]. The subgroup $\mathcal{F}_{2}$ is defined to be the kernel of $C$. We do not consider $C$ or $\mathcal{F}_{2}$ in this paper, this will be done in later work.

We now need to review the definitions of the isomorphisms $\xi: K_{i} \rightarrow \mathfrak{K}_{i}$, for $i=0,1$ from $[\mathbf{6}]$, but refer the reader to $[6]$ for all details.

We recall the classical definition of $\left.K_{1}(T)\right)$ as $K_{1}(T)^{\text {det }}$, for a ring $T$. One can define the group $K_{1}(T)$ to be the free abelian group on the set of pairs $(P, \alpha)$, where $P$ is a finitely generated projective module and $\alpha$ is an automorphism of $P$, modulo the subgroup generated by the elements

- $(P, \alpha)+(P, \beta)-(P, \alpha \beta)$

- $(X, \beta)-(P, \alpha)-(Q, \gamma)$, whenever

$$
\begin{array}{ccccccccc}
0 & \rightarrow & P & \stackrel{f}{\rightarrow} & X & \stackrel{g}{\rightarrow} & Q & \rightarrow & 0 \\
& \alpha \downarrow & & \beta \downarrow & & \gamma \downarrow & & \\
0 & \rightarrow & P & \stackrel{f}{\rightarrow} & X & \stackrel{g}{\rightarrow} & Q & \rightarrow & 0
\end{array}
$$

is a commutative diagram of $T$-modules with exact rows and $\alpha, \beta, \gamma$ are automorphisms.

Using this notation, it is well-known that every element of $K_{1}(T)^{\text {det }}$ has a representative of the form $\left(T^{n}, A\right)$, where $A$ is an invertible $n \times n$-matrix with entries in $T$.

Let $K_{0}(T)$ denote the classically defined $K_{0}$; if $Z$ is a finitely generated projective $T$-module, let $[Z]$ denote the equivalence class of the isomorphism class of $Z$ in $K_{0}(T)$.

The isomorphism $\xi: K_{0}(T) \rightarrow \pi_{0}(G T)$ is defined by

$$
\xi([Z])=\left[t\left(\iota_{Z}\right)\right]
$$

Now, if $\alpha: P \rightarrow P$ is an automorphism of a finitely generated projective $T$-module, there is an element $x(\alpha) \in G T_{1}$ defined by

$$
x(\alpha)=t\left(q_{P} \mid \alpha\right) t\left(\iota_{P} \mid \alpha\right)^{-1} .
$$

The isomorphism $\xi: K_{1}(T) \rightarrow \pi_{1}(G T)$ is defined by

$$
\xi([P, \alpha])=[x(\alpha)] .
$$

5.3.3. $j\left(\mathcal{F}_{0}\right) \subseteq F_{0,0}$

The composite

$$
\pi_{0}\left(\overleftarrow{\mathcal{I}}_{\lim } G \Re\right) \rightarrow \pi_{0}(\operatorname{Tot}(\mathcal{I}, G \Re)) \stackrel{p_{*}}{\rightarrow}{\underset{\mathcal{I}}{\mathcal{I}}}_{\lim _{0}} \pi_{\mathfrak{R}} \doteq H^{0}\left(\mathcal{I}, \pi_{0} G \Re\right)
$$

is equal to the natural homomorphism

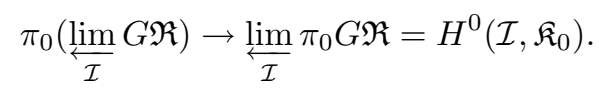

Note that $p_{*}$ is the map at the $\pi_{0}$ level induced by the fibration $p:$ Tot $\rightarrow$ Tot $_{0}$. 
The desired conclusion now follows from the naturality property of the isomorphism $\xi$, and the universal properties of direct limits. But, one may give an "element-wise" proof as follows.

In all of our applications, inverse limits of a functor can be realized as subsets of the direct product, calculated in the range category of the functor, of the values of the functor on the object set of the domain category of the functor. We will use "tuple" notation for elements of the direct product. For example, if the value of a functor on the object $v$ of the domain category is $A_{v}$, then an element of the direct product $\prod_{v} A_{v}$ will be denoted by $\left(a_{v}\right)_{v}$, where $a_{v} \in A_{v}$, for every object $v$.

Thus, if $[P] \in K_{0}\left(\lim _{\mathcal{I}} \mathfrak{R}\right)$,

$$
p_{*} j([P])=\left(\left[t\left(\iota_{P(v)}\right)\right]\right)_{v},
$$

also, considering the isomorphism

$$
H^{0}\left(\mathcal{I}, \mathcal{K}_{0}\right) \stackrel{\xi}{\rightarrow} H^{0}\left(\mathcal{I}, \mathfrak{K}_{0}\right)
$$

we see, using the above formula for $p_{*} j([P])$ and the definition of $\xi$, that

$$
\xi(J([P]))=\xi\left(([P(v)])_{v}\right)=p_{*} j([P]) .
$$

By definition, $\operatorname{ker} J=\mathcal{F}_{0}$, which we now see is equal to ker $k_{1} j$. Also, by definition, $\operatorname{ker} p_{*}=F_{0,0}$. Thus,

$$
j\left(\mathcal{F}_{0}\right) \subseteq F_{0,0}
$$

5.3.4. $j\left(\mathcal{F}_{1}\right) \subseteq F_{1,0}$

Consider the homomorphisms

$$
\mathcal{F}_{0} \stackrel{\left.j\right|_{\mathcal{F}_{0}}}{\longrightarrow} F_{0,0} \stackrel{b_{1}}{\longrightarrow} H^{1}\left(\mathcal{I}, \mathfrak{K}_{1}\right) \stackrel{\xi}{\longleftarrow} H^{1}\left(\mathcal{I}, \mathcal{K}_{1}\right) \stackrel{B}{\longleftarrow} \mathcal{F}_{0} .
$$

We know that $b_{1}$ is the composite

$$
F_{0,0} \rightarrow E_{\infty}^{1,1} \subseteq E_{2}^{1,1} \stackrel{\beta_{1}}{\longrightarrow} H^{1}\left(\mathcal{I}, \pi_{1} G \Re\right) ;
$$

the isomorphism

$$
\left.\beta_{1}: E_{2}^{1,1} \rightarrow H^{1}\left(\mathcal{I}, \pi_{1} G \Re\right)\right)
$$

is that described in Section 4.2.2. We will prove that $\left.b_{1} j\right|_{\mathcal{F}_{0}}$ is equal to the map $-\xi B$. Then,

$$
F_{1,0} \doteq \operatorname{ker}\left(F_{0,0} \rightarrow E_{\infty}^{1,1}\right)=\operatorname{ker}\left(F_{0,0} \stackrel{b_{1}}{\rightarrow} H^{1}\left(\mathcal{I}, \pi_{1} G \Re\right)\right) .
$$

So, since $\mathcal{F}_{1}=\operatorname{ker} B$ and $\left.b_{1} j\right|_{\mathcal{F}_{0}}=-\xi B, j\left(\mathcal{F}_{1}\right) \subseteq \operatorname{ker} b_{1}=F_{1,0}$.

Now, by definition, $F_{0,0}$ is the kernel of the homomorphism

$$
\pi_{0}(\operatorname{Tot}(\mathcal{I}, G \mathfrak{R})) \rightarrow \pi_{0}\left(\operatorname{Tot}_{0}(\mathcal{I}, G \mathfrak{R})\right) \doteq C^{0}\left(\mathcal{I}, \pi_{0} G \mathfrak{R}\right) .
$$

Let

$$
[\gamma] \in F_{0,0} \subseteq \pi_{0}(\operatorname{Tot}(\mathcal{I}, G \Re)) .
$$

Let $\gamma_{i}$ denote the image of $\gamma$ under the homomorphism $\operatorname{Tot}(\mathcal{I}, G \Re) \rightarrow \operatorname{Tot}_{i}(\mathcal{I}, G \Re)$. Recall that $F_{i}$ is the kernel of the homomorphism $\operatorname{Tot}_{i}(\mathcal{I}, G \Re) \rightarrow \operatorname{Tot}_{i-1}(\mathcal{I}, G \Re)$. 
Since $\left[\gamma_{0}\right]=[1]$ in the group $\pi_{0}\left(\operatorname{Tot}_{0}(\mathcal{I}, G \Re)\right)=C^{0}\left(\mathcal{I}, \pi_{0} G \Re\right)$, using the long exact sequence of the fibration $\operatorname{Tot}_{1}(\mathcal{I}, G \Re) \rightarrow \operatorname{Tot}_{0}(\mathcal{I}, G \Re)$, there exists $[\tau] \in \pi_{0}\left(F_{1}\right)$ such that $[\tau]=\left[\gamma_{1}\right]$ in $\pi_{0}\left(\operatorname{Tot}_{1}(\mathcal{I}, G \Re)\right)$.

From now on, we often make the abbreviations

$$
\operatorname{Tot}_{i}(\mathcal{I}, G \Re)=\operatorname{Tot}_{i}, \quad \text { and } \quad C^{*}(\mathcal{I}, G \Re)=C^{*} .
$$

More precisely, $\tau$ is obtained as follows, using the description of Tot $_{1}$ given in Section 2.3 of this paper. First, since $\left[\gamma_{0}\right]=[1]$, there exists an element $\tilde{\gamma}_{0} \in\left(\overline{T o t_{0}}\right)_{1} \subseteq C_{1}^{0}$ such that $d_{1}\left(\tilde{\gamma}_{0}\right)=\gamma_{0}$. (The $d_{1}$ of this discussion is the indicated face map of the simplicial group $\operatorname{Tot}_{0}=C^{0}$.) Using Section 2.3, we see that there exists an element $\sigma_{\tilde{\gamma}_{0}} \in\left(\overline{\operatorname{Hom}\left(\Delta^{1}, C^{1}\right)}\right)_{1}$ such that $\left(\tilde{\gamma}_{0}, \sigma_{\tilde{\gamma}_{0}}\right)$ is an element of

$$
\left(\overline{\operatorname{Tot}_{1}}\right)_{1} \subseteq \overline{C_{1}^{0}} \times \overline{\operatorname{Hom}\left(\Delta^{1}, C^{1}\right)_{1}}
$$

and maps to $\tilde{\gamma_{0}}$ via the fibration $\operatorname{Tot}_{1} \rightarrow \operatorname{Tot}_{0}=C^{0}$. Then,

$$
d_{1}\left(\tilde{\gamma}_{0}, \sigma_{\tilde{\gamma}_{0}}\right)^{-1} \gamma_{1} \in\left(F_{1}\right)_{0} \subseteq\left(\operatorname{Tot}_{1}\right)_{0} \subseteq \overline{C_{0}^{0}} \times \overline{\operatorname{Hom}\left(\Delta^{1}, C^{1}\right)_{0}}=C_{0}^{0} \times C_{1}^{1},
$$

and we set

$$
\tau=d_{1}\left(\tilde{\gamma}_{0}, \sigma_{\tilde{\gamma}_{0}}\right)^{-1} \gamma_{1}=\left(1, \tau_{1}\right) \in C_{0}^{0} \times C_{1}^{1}
$$

As we already know, the homotopy class of $\tau$ is independent of the choices made.

By definition, $[\tau] \in Z_{2}^{1,1} \doteq\left(\operatorname{ker} \pi_{0}\left(F_{1}\right) \rightarrow \pi_{0}\left(\operatorname{Tot}_{1}\right) / \pi_{0}^{1}\left(\operatorname{Tot}_{1}\right)\right)$, since $[\tau]=\left[\gamma_{1}\right]$ in $\pi_{0}\left(\operatorname{Tot}_{1}\right)$ is clearly the image of $\left[\gamma_{2}\right] \in \pi_{0}\left(\operatorname{Tot}_{2}\right)$.

Now, $b_{1}([\gamma])$ is equal to the cohomology class of $\left[\tau_{1}\right] \in Z^{1}\left(\mathcal{I}, \pi_{1} G \mathfrak{R}\right)$.

Next, assume that $[\gamma]$ is in the image of $\left.j\right|_{\mathcal{F}_{0}}$.

Identify $\pi_{0}\left(\operatorname{Tot}_{0}(\mathcal{I}, G \Re)\right)$ with the quotient group

$$
C^{0}\left(\mathcal{I}, G \Re_{0}\right) / \operatorname{im} \bar{d}_{1},
$$

where

$$
\bar{d}_{1}: C^{0}\left(\mathcal{I}, G \Re_{1} \cap \operatorname{ker} d_{0}\right) \rightarrow C^{0}\left(\mathcal{I}, G \Re_{0}\right)
$$

is induced by $d_{1}$.

Suppose that $[\gamma]=j\left([P]-\left[R^{n}\right]\right)=j\left(-\left[R^{n}\right]+[P]\right)$, where

$$
[P]-\left[R^{n}\right] \in \operatorname{ker} J \subseteq K_{0}(R) .
$$

Thus, $\gamma_{0} \in \pi_{0}\left(\operatorname{Tot}_{0}\right)$ is the homotopy class of the element

$$
v \mapsto t\left(\iota_{Q(v)}\right)^{-1} t\left(\iota_{P(v)}\right) \in G \Re(v)_{0}
$$

in $C^{0}\left(\mathcal{I}, G \Re_{0}\right)$; abusing notation somewhat, we will also denote this element of $C^{0}\left(\mathcal{I}, G \Re_{0}\right)$ by $\gamma_{0}$. 
We can write down $\gamma_{1}$ using Theorem 2.4.1: $\gamma_{1} \in \pi_{0}\left(\right.$ Tot $\left._{1}\right)$ may be identified with the homotopy class of an ordered pair $\left(\gamma_{0}, Z\right)$ in the simplicial group

$$
C_{0}^{0} \times \operatorname{Hom}\left(\Delta^{1}, C^{1}\right)_{0}=C_{0}^{0} \times C_{1}^{1},
$$

where $Z$ has the following properties:

$$
d_{1} Z=d^{1} \gamma_{0}, d_{0} Z=d^{0} \gamma_{0}, s^{0} Z=s_{0} \gamma_{0}
$$

Such a $Z \in C_{1}^{1}$ can be defined by

$$
v \stackrel{g}{\rightarrow} w \mapsto t\left(1 \mid \iota_{Q(w)}\right)^{-1} t\left(1 \mid \iota_{P(w)}\right) .
$$

Using the definitions,

$$
d_{1} Z(v \stackrel{g}{\rightarrow} w)=t\left(\iota_{Q(w)}\right)^{-1} t(1) t(1)^{-1} t\left(\iota_{P(w)}\right)=d_{0} Z(v \stackrel{g}{\rightarrow} w),
$$

and since $g_{*}\left(t\left(\iota_{Q(v)}\right)^{-1} t\left(\iota_{P(v)}\right)\right)=t\left(\iota_{Q(w)}\right)^{-1} t\left(\iota_{P(w)}\right)$,

$$
d^{1} \gamma_{0}(v \stackrel{g}{\rightarrow} w)=g_{*}\left(\gamma_{0}(v)\right)=t\left(\iota_{Q(w)}\right)^{-1} t\left(\iota_{P(w)}\right)=d^{0} \gamma_{0}(v \stackrel{g}{\rightarrow} w),
$$

as well. Finally,

$$
s^{0} Z(v)=Z(v \stackrel{i d}{\rightarrow} v)=t\left(1 \mid \iota_{Q(v)}\right)^{-1} t\left(1 \mid \iota_{P(v)}\right)=s_{0} \gamma_{0}(v),
$$

for every $v$. Again, we will abuse notation and refer to the element $\left(\gamma_{0}, Z\right)$ described above as $\gamma_{1}$ (confusing an element with its homotopy class).

Now, let us bring in the assumption that $[P]-\left[R^{n}\right] \in \operatorname{ker} J \subseteq K_{0}(R)$. We may assume that there exist isomorphisms of $\mathfrak{R}(v)$-modules

$$
\theta_{v}: j_{*}^{v}(P) \doteq P(v) \rightarrow \mathfrak{R}(v)^{n} \doteq Q(v)
$$

for every $v \in \mathcal{I}$.

This allows us to define an element $\tilde{\gamma}_{0} \in C^{0}\left(\mathcal{I}, G \Re_{1}\right)$ by

$$
v \mapsto t\left(q_{P(v)} \mid \theta_{v}\right) t\left(\iota_{P(v)} \mid \theta_{v}\right)^{-1} \in(G \Re(v))_{1},
$$

for every $v \in \mathcal{I}$. Note that $\tilde{\gamma}_{0} \in\left(\overline{\operatorname{Tot}}_{0}\right)_{1}=\overline{C^{0}\left(\mathcal{I}, G \Re_{1}\right)}$, since $d_{0} \tilde{\gamma}_{0}=1$. Also, for every $v \in \mathcal{I}$

$$
d_{1}\left(\tilde{\gamma}_{0}(v)\right)=t\left(q_{P(v)}\right)^{-1} t\left(\theta_{v} q_{P(v)}\right) t\left(\theta_{v} \iota_{P(v)}\right)^{-1} t\left(\iota_{P(v)}\right),
$$

which equals

$$
t\left(\iota_{Q(v)}\right)^{-1} t\left(\iota_{P(v)}\right)
$$

therefore $d_{1} \tilde{\gamma}_{0}=\gamma_{0}$.

Calculating, always directly from the definitions, we see that, if $v \stackrel{g}{\rightarrow} w \in \mathcal{N} \mathcal{I}_{1}$,

$$
d^{1} \tilde{\gamma}_{0}(v \stackrel{g}{\rightarrow} w)=g_{*}\left(\tilde{\gamma}_{0}(v)\right)=t\left(q_{P(w)} \mid g_{*} \theta_{v}\right) t\left(\iota_{P(w)} \mid g_{*} \theta_{v}\right)^{-1}
$$

and

$$
d^{0} \tilde{\gamma}_{0}(v \stackrel{g}{\rightarrow} w)=t\left(q_{P(w)} \mid \theta_{w}\right) t\left(\iota_{P(w)} \mid \theta_{w}\right)^{-1} .
$$

Now, given a morphism $v \stackrel{g}{\rightarrow} w$ in $\mathcal{I}$, define an element $B(v \stackrel{g}{\rightarrow} w) \in(G \Re(w))_{2}$ by

$$
\begin{aligned}
B(v \stackrel{g}{\rightarrow} w)= & t\left(1\left|q_{P(w)}\right| \theta_{w}\right) t\left(q_{Q(w)}\left|\theta_{w}^{-1}\right| \theta_{w}\right)^{-1} t\left(q_{Q(w)}\left|\theta_{w}^{-1}\right| g_{*} \theta_{v}\right) \\
& \cdot t\left(\iota_{Q(w)}\left|\theta_{w}^{-1}\right| g_{*} \theta_{v}\right)^{-1} t\left(\iota_{Q(w)}\left|\theta_{w}^{-1}\right| \theta_{w}\right) t\left(1\left|\iota_{P(w)}\right| \theta_{w}\right)^{-1} .
\end{aligned}
$$


Compute that

$$
d_{0}(B(v \stackrel{g}{\rightarrow} w))=t\left(q_{P(w)} \mid \theta_{w}\right) t\left(\iota_{P(w)} \mid \theta_{w}\right)^{-1}=d^{0} \tilde{\gamma}_{0}(v \stackrel{g}{\rightarrow} w),
$$

and

$$
\begin{aligned}
d_{1}(B(v \stackrel{g}{\rightarrow} w))= & t\left(q_{P(w)} \mid \theta_{w}\right) t\left(q_{P(w)} \mid \theta_{w}\right)^{-1} t\left(q_{P(w)} \mid g_{*} \theta_{v}\right) \\
& \cdot t\left(\iota_{P(w)} \mid g_{*} \theta_{v}\right)^{-1} t\left(\iota_{P(w)} \mid \theta_{w}\right) t\left(\iota_{P(w)} \mid \theta_{w}\right)^{-1} \\
= & t\left(q_{P(w)} \mid g_{*} \theta_{v}\right) t\left(\iota_{P(w)} \mid g_{*} \theta_{v}\right)^{-1}=d^{1} \tilde{\gamma}_{0}(v \stackrel{g}{\rightarrow} w) .
\end{aligned}
$$

Furthermore,

$$
\begin{aligned}
d_{2}(B(v \stackrel{g}{\rightarrow} w))= & t\left(q_{Q(w)} \mid \theta_{w}^{-1}\right) t\left(q_{Q(w)} \mid \theta_{w}^{-1}\right)^{-1} t\left(q_{Q(w)} \mid g_{*} \theta_{v} \theta_{w}^{-1}\right) t\left(\iota_{Q(w)} \mid g_{*} \theta_{v} \theta_{w}^{-1}\right)^{-1} \\
& \cdot t\left(\iota_{Q(w)} \mid \theta_{w}^{-1}\right) t\left(\iota_{Q(w)} \mid \theta_{w}^{-1}\right)^{-1} t\left(1 \mid \iota_{Q(w)}\right)^{-1} t\left(1 \mid \iota_{P(w)}\right) \\
= & t\left(q_{Q(w)} \mid g_{*} \theta_{v} \theta_{w}^{-1}\right) t\left(\iota_{Q(w)} \mid g_{*} \theta_{v} \theta_{w}^{-1}\right)^{-1} Z \doteq x\left(g_{*} \theta_{v} \theta_{w}^{-1}\right) Z .
\end{aligned}
$$

Finally,

$$
s^{0} B(v)=B(v \stackrel{i d}{\rightarrow} v)=t\left(1\left|q_{P(v)}\right| \theta_{v}\right) t\left(1\left|\iota_{P(v)}\right| \theta_{v}\right)^{-1}=s_{0} \tilde{\gamma}_{0},
$$

and

$$
s^{0} d_{2} B(v)=d_{2} B(v \stackrel{i d}{\rightarrow} v)=t\left(1 \mid \iota_{Q(v)}\right)^{-1} t\left(1 \mid \iota_{P(v)}\right)=s_{0}\left(t\left(\iota_{Q(v)}\right)^{-1} t\left(\iota_{P(v)}\right)\right)=s_{0} \gamma_{0} .
$$

Now, define $A=s_{1} d_{1} B$, and consider the triple $\left(\tilde{\gamma}_{0}, B, A\right) \in \overline{C_{1}^{0}} \times C_{2}^{1} \times C_{2}^{1}$.

We have already shown that

$$
d_{0} B=d^{0} \tilde{\gamma}_{0}, d_{1} B=d^{1} \tilde{\gamma}_{0}, s^{0} B=s_{0} \tilde{\gamma}_{0}
$$

Also,

$$
\begin{aligned}
& d_{1} B=d_{1} s_{1} d_{1} B=d_{1} A, d_{2} A=d_{2} s_{1} d_{1} B=d_{1} B=d^{1} \tilde{\gamma}_{0}, \\
& d_{0} A=d_{0} s_{1} d_{1} B=s_{0} d_{0} d_{1} B=s_{0} d_{0} d_{0} B=s_{0} d^{0} d_{0} \tilde{\gamma}_{0}=1
\end{aligned}
$$

and

$$
s^{0} A=s^{0} s_{1} d_{1} B=s^{0} s_{1} d^{1} \tilde{\gamma}_{0}=s_{1} \tilde{\gamma}_{0} .
$$

Thus, $\left(\tilde{\gamma}_{0}, B, A\right)$ defines an element of $\left(\overline{\operatorname{Tot}_{1}}\right)_{1}$, using Theorem 2.4; note that this element projects to $\tilde{\gamma}_{0}$ under the fibration Tot $_{1} \rightarrow$ Tot $_{0}$. Continuing to compute, using Theorem 2.4,

$$
d_{1}\left(\tilde{\gamma}_{0}, B, A\right)=\left(d_{1} \tilde{\gamma}_{0}, d_{2} B\right)
$$

SO

$$
d_{0}\left(\tilde{\gamma}_{0}, A, B\right)^{-1} \gamma_{1}=\left(d_{1} \tilde{\gamma}_{0}^{-1} \gamma_{0}, d_{2} B^{-1} Z\right)=\left(1, d_{2} B^{-1} Z\right)
$$

To conclude, we have shown that

$$
b_{1} j\left([P]-\left[R^{n}\right]\right)=\text { the cohomology class of }\left[d_{2} B^{-1} Z\right] \in Z^{1}\left(\mathcal{I}, \pi_{1} G \Re\right) ;
$$

by inspection, the element

$$
\left[d_{2} B^{-1} Z\right]=\left[Z^{-1} X Z\right]
$$


where $X \in C_{1}^{1}$ is defined by

$$
X(v \stackrel{g}{\rightarrow} w)=x\left(g_{*} \theta_{v} \theta_{w}^{-1}\right)^{-1} .
$$

Now,

$$
\left[Z^{-1} X Z\right]=[X] \in Z^{1}\left(\mathcal{I}, \pi_{1} G \Re\right),
$$

since $\pi_{1} G T$ is central in $G T_{1} / B G T_{1}$, for any commutative ring $T$.

Thus,

$$
b_{1} j\left([P]-\left[R^{n}\right]\right)=\text { the cohomology class of }[X] \in Z^{1}\left(\mathcal{I} \pi_{1} G \Re\right),
$$

which is, by the results of [6], equal to the cohomology class of

$$
-\xi B\left([P]-\left[R^{n}\right]\right) .
$$

\section{A simple example}

In this example, the category $\mathcal{I}$ is the category associated to a poset $\mathcal{I}$ which has only three elements, call them 1,2 and 12; the only nonreflexive inequalities are $1<12,2<12$. In this case a presheaf of (looped, since we would like to assume all homotopy is abelian) simplicial groups on $\mathcal{I}$ is just a diagram, call it $\mathfrak{F}$,

$$
\begin{array}{ccc} 
& \left(G_{1}, \theta_{1}, H_{1}\right) \\
& \downarrow f \\
& & \\
\left(G_{2}, \theta_{2}, H_{2}\right) & \stackrel{g}{\rightarrow} & \left(G_{12}, \theta_{12}, H_{12}\right)
\end{array}
$$

of simplicial groups and simplicial group homomorphisms.

The construction of the Bousfield-Kan spectral sequence (in this simple case, it is not really necessary to use the extended spectral sequence) shows that there exists a filtration

$$
1=F_{1, j} \subseteq F_{0, j} \subseteq \pi_{j}(\operatorname{Tot}(\mathcal{I}, \mathfrak{F}))=F_{-1, j},
$$

of $\pi_{j}(\operatorname{Tot}(\mathcal{I}, \mathfrak{F}))=\pi_{j}\left(\operatorname{Tot}_{1}(\mathcal{I}, \mathfrak{F})\right)$, for every $j \geqslant 0$. This filtration has the property that

- $\pi_{j}\left(\operatorname{Tot}(\mathcal{I}, \mathfrak{F}) / F_{0, j} \cong H^{0}\left(\mathcal{I}, \pi_{j} \mathfrak{F}\right) \cong E_{2}^{0, j}=E_{\infty}^{0, j}\right.$, for every $j \geqslant 0$, and

- $F_{0, j} \cong H^{1}\left(\mathcal{I}, \pi_{j+1} \mathfrak{F}\right) \cong E_{2}^{1, j+1}=E_{\infty}^{1, j+1}$, for every $j \geqslant 0$.

We can compute the cohomology groups in this simple example.

By definition, for every $j \geqslant 0$,

$$
H^{0}\left(\mathcal{I}, \pi_{j} \mathfrak{F}\right)=\left\{(a, b) \in \pi_{j}\left(G_{1}\right) \times \pi_{j}\left(G_{2}\right) \mid f_{*}(a)=g_{*}(b)\right\} .
$$

Also, by definition,

$$
H^{1}\left(\mathcal{I}, \pi_{j+1} \mathfrak{F}\right)=\left(\pi_{j+1}\left(G_{12}\right) \times \pi_{j+1}\left(G_{12}\right)\right) / N,
$$

where $N=\left\{\left(f_{*}(a) c^{-1}, g_{*}(b) c^{-1}\right) \mid(a, b, c) \in \pi_{j+1}\left(G_{1}\right) \times \pi_{j+1}\left(G_{2}\right) \times \pi_{j+1}\left(G_{12}\right)\right\}$.

This last group is isomorphic to $\pi_{j+1}\left(G_{12}\right) / K$, where

$$
K=\left\{f_{*}(a) g_{*}(b)^{-1} \mid(a, b) \in \pi_{j+1}\left(G_{1}\right) \times \pi_{j+1}\left(G_{2}\right)\right\}
$$

via the homomorphism induced by $\pi_{j+1}\left(G_{12}\right) \times \pi_{j+1}\left(G_{12}\right) \rightarrow \pi_{j+1}\left(G_{12}\right)$ given by $(x, y) \mapsto x y^{-1}$; this is a homomorphism since the groups $\pi_{j+1}$ are abelian for $j \geqslant 0$. 
Using these identifications, and the properties of the filtration on $\operatorname{Tot}(\mathcal{I}, \mathfrak{F})$, we obtain a long exact sequence (which is functorial on the appropriate domain category) of homotopy groups:

$$
\begin{aligned}
\cdots \rightarrow \pi_{j+1}\left(G_{12}\right) \stackrel{\hat{\delta}}{\rightarrow} \pi_{j}(\operatorname{Tot}(\mathcal{I}, \mathfrak{F})) \stackrel{V}{\rightarrow} \pi_{j}\left(G_{1}\right) \times \pi_{j}\left(G_{2}\right) & \\
\stackrel{A}{\rightarrow} & \pi_{j}\left(G_{12}\right) \rightarrow \pi_{j-1}(\operatorname{Tot}(\mathcal{I}, \mathfrak{F})) \rightarrow \cdots,
\end{aligned}
$$

where the sequence ends at $\pi_{0}$ :

$$
\cdots \rightarrow \pi_{1}\left(G_{12}\right) \stackrel{\hat{\delta}}{\rightarrow} \pi_{0}(\operatorname{Tot}(\mathcal{I}, \mathfrak{F})) \stackrel{V}{\rightarrow} \pi_{0}\left(G_{1}\right) \times \pi_{0}\left(G_{2}\right) \stackrel{A}{\rightarrow} \pi_{0}\left(G_{12}\right) .
$$

The homomorphism $A$ is defined by $A(a, b)=f_{*}(a) g_{*}(b)^{-1}$.

The homomorphism $\hat{\delta}$ is defined by the composite

$$
\begin{aligned}
& \pi_{j+1}\left(G_{12}\right) \rightarrow\left(\pi_{j+1}\left(G_{12}\right) \times \pi_{j+1}\left(G_{12}\right)\right) / K \\
& \cong H^{1}\left(\mathcal{I}, \pi_{j+1} \mathfrak{F}\right) \cong E_{2}^{1, j+1} \cong E_{\infty}^{1, j+1} \subseteq \pi_{j}\left(\text { Tot }_{1}\right),
\end{aligned}
$$

and for $j \geqslant 0$, the homomorphism $V$ is defined by the composite

$$
\pi_{j}\left(\operatorname{Tot}_{1}\right) \rightarrow H^{0}\left(\mathcal{I}, \pi_{j} \mathfrak{F}\right) \subseteq \pi_{j}\left(G_{1}\right) \times \pi_{j}\left(G_{2}\right) .
$$

A special case of the above example occurs when, in the diagram $\mathfrak{F}$, the group $G_{2}$ is weakly equivalent to the identity group. Then, the above exact sequence becomes

$$
\cdots \rightarrow \pi_{j+1}\left(G_{12}\right) \rightarrow \pi_{j}(\operatorname{Tot}(\mathcal{I}, \mathfrak{F})) \rightarrow \pi_{j}\left(G_{1}\right) \stackrel{f_{*}}{\rightarrow} \pi_{j}\left(G_{12}\right) \rightarrow \pi_{j-1}(\operatorname{Tot}(\mathcal{I}, \mathfrak{F})) \rightarrow \cdots
$$

Thus, one recovers the fact that $\operatorname{Tot}(\mathcal{I}, \mathfrak{F})$ is the homotopy fibre of the map $f$.

If $F$ is the kernel of the homomorphism $f$, then there is an augmentation $\nu: F \rightarrow C^{0}(\mathcal{I}, \mathfrak{F})$ and a natural homomorphism of simplicial groups $F \rightarrow \operatorname{Tot}(\mathcal{I}, \mathfrak{F})$. If $G_{2}$ is weakly equivalent to the identity group, and $f$ is a fibration, then we see that the homomorphism $F \rightarrow \lim _{\mathcal{I}} \mathfrak{F} \rightarrow \operatorname{Tot}(\mathcal{I}, \mathfrak{F})$ is a weak equivalence, as expected. If $f$ is not a fibration, then $F \rightarrow \operatorname{Tot}(\mathcal{I}, \mathfrak{F})$ of course may not be a weak equivalence; a very simple example of this is obtained by setting $G_{1}=G_{2}=1$, and letting $G_{12}$ be any simplicial group not weakly equivalent to a point. Then, $F=1$, but $\operatorname{Tot}(\mathcal{I}, \mathfrak{F})$ is weakly equivalent to $\Omega G_{12}$.

In general, under the right conditions (for example, if at least one of the maps $f, g$ is a fibration $([\mathbf{4}, \mathrm{XI}, 4.1,4.4]))$, the natural homomorphism $\phi: \lim _{\mathcal{I}} \mathfrak{F} \rightarrow \operatorname{Tot}(\mathcal{I}, \mathfrak{F})$ is a weak equivalence; indeed, one has the following theorem:

Theorem 6.1 ([4, XI, 4.1]). If $\mathcal{I}$ is the poset of this section, and the map $g: G_{2} \rightarrow G_{12}$ is a fibration of simplicial groups, then the natural homomorphism $\phi: \lim _{\mathcal{I}} \mathfrak{F} \rightarrow \operatorname{Tot}(\mathcal{I}, \mathfrak{F})$ is a weak equivalence, and there is a natural long exact sequence of groups

$$
\begin{aligned}
\cdots \rightarrow \pi_{j+1}\left(G_{12}\right) \stackrel{\mathcal{D}=\hat{\delta} \circ \phi_{*}^{-1}}{\longrightarrow} \pi_{j}(\underbrace{}_{\overleftarrow{\mathcal{I}}}(\lim \mathfrak{F}) \stackrel{\mathcal{P}=V \circ \phi_{*}}{\longrightarrow} & \pi_{j}\left(G_{1}\right) \times \pi_{j}\left(G_{2}\right) \\
& \stackrel{A}{\rightarrow} \pi_{j}\left(G_{12}\right) \stackrel{\mathcal{D}}{\rightarrow} \pi_{j-1}\left(\overleftarrow{\lim }_{\mathcal{I}} \mathfrak{F}\right) \rightarrow \cdots,
\end{aligned}
$$

where the exact sequence ends with $\pi_{0}$. 
To begin a brief sketch of the proof, note that since $g$ is a fibration, there is a long exact sequence in homotopy, with connecting homomorphisms $\delta_{2}: \pi_{j+1}\left(G_{12}\right) \rightarrow$ $\pi_{j}(\operatorname{ker} g)$ for every $j \geqslant 0$. Let $\iota$ be the inclusion of $\operatorname{ker} g$ into $G_{1} \times{ }_{G_{12}} G_{2}$, and let $p: G_{1} \times_{G_{12}} G_{2} \rightarrow G_{1}$ and $q: G_{1} \times_{G_{12}} G_{2} \rightarrow G_{2}$ be the natural projections.

Consider the following diagram, for $t \geqslant 0$ :

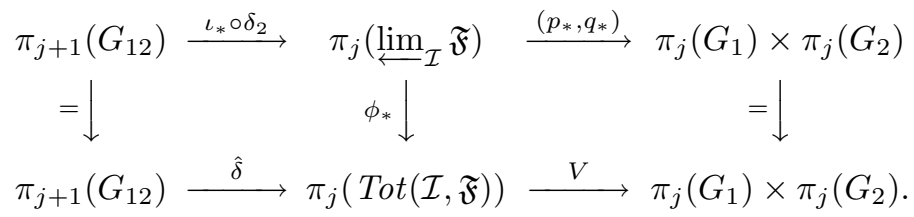

Since this diagram commutes up to sign, one can make the desired conclusion.

Also, alternate descriptions of the maps $\mathcal{D}, \mathcal{P}$ the long exact sequence of the theorem are

$$
\begin{aligned}
& \mathcal{D}=\iota_{*} \circ \delta_{2}, \\
& \mathcal{P}=\left(p_{*}, q_{*}\right) .
\end{aligned}
$$

\subsection{The "Mayer-Vietoris" sequence in $K$-theory}

Let $\mathcal{I}$ be the same simple one-dimensional poset of the previous section.

A presheaf of rings $\mathfrak{R}$ on $\mathcal{I}$ is a diagram of rings

$$
\begin{array}{lll} 
& & R_{1} \\
& & \downarrow f \\
R_{2} \quad \stackrel{g}{\rightarrow} & R_{12} ;
\end{array}
$$

applying the functor $G$ gives a diagram (presheaf) of looped simplicial groups $G \Re$

$$
\begin{array}{ccc} 
& & G R_{1} \\
& & \downarrow G f \\
G R_{2} \stackrel{G g}{\longrightarrow} & G R_{12} .
\end{array}
$$

For this poset $\mathcal{I}$ and the particular cosimplicial simplicial group $C(\mathcal{I}, G \Re)$, the long exact sequence of the previous section may be rewritten as follows:

$$
\cdots \rightarrow \pi_{t+1}\left(G R_{12}\right) \rightarrow \pi_{t}(\operatorname{Tot}(\mathcal{I}, G \Re)) \rightarrow \pi_{t}\left(G R_{1}\right) \times \pi_{t}\left(G R_{2}\right) \stackrel{A}{\rightarrow} \pi_{t}\left(G R_{12}\right) \rightarrow \cdots,
$$

where the sequence ends at $\pi_{0}$ :

$$
\cdots \rightarrow \pi_{1}\left(G R_{12}\right) \rightarrow \pi_{0}(\operatorname{Tot}(\mathcal{I}, G \Re)) \rightarrow \pi_{0}\left(G R_{1}\right) \times \pi_{0}\left(G R_{2}\right) \stackrel{A}{\rightarrow} \pi_{0}\left(G R_{12}\right) .
$$

Now, as we have seen, if one of $G f, G g$ is a fibration, then the natural map of simplicial groups

$$
G R_{1} \times_{G R_{12}} G R_{2}={\underset{\mathcal{I}}{\lim }}_{\operatorname{li}} G \Re \rightarrow \operatorname{Tot}(\mathcal{I}, G \Re)
$$

induces isomorphisms on all homotopy groups.

We assume that $G g$ is a fibration, and that $g$ is surjective.

Let $L$ be the simplicial group $G R_{1} \times_{G R_{12}} G R_{2}$. 
Let $R$ be the pullback of the diagram $\mathfrak{R}$; indeed, by definition,

$$
R=R_{1} \times R_{12} R_{2}=\underbrace{\lim }_{\mathcal{I}} \Re
$$

We examine the end of this long exact sequence; it is the top row in the diagram below:

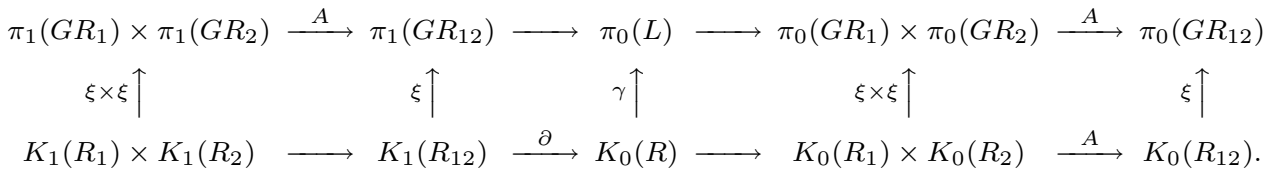

The bottom row is Milnor's exact sequence in classical $K$-theory, constructed in $[\mathbf{1 3}]$.

The maps $\xi$ are the isomorphisms defined in [6], and reviewed in Section 5.3. The left- and right-most squares commute because of the naturality of these maps.

The center vertical map $\gamma$ above is the composite $\nu_{1 *} \circ \xi$, where $\nu_{1}$ is the natural map of simplicial groups $\nu_{1}: G R \rightarrow L=H^{0}(\mathcal{I}, G \Re)$. The third-from-left square in the diagram above commutes because of the naturality of $\xi$.

We now investigate the second-from-left square in the diagram above:

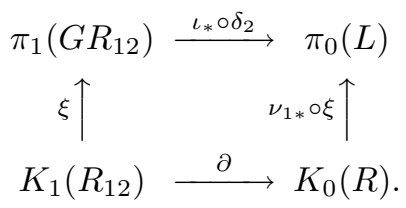

Lemma 6.2. Assuming that the map $g$ above is surjective, the square

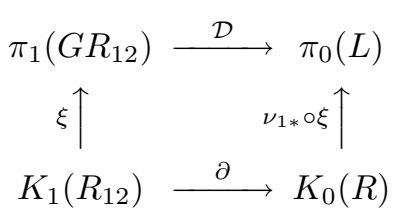

described above is a commutative square.

In order to give the proof of this lemma, we must recall the construction of $\partial$ (from $[\mathbf{1 3}]$ ).

Suppose that $A$ is an invertible $n \times n$ matrix with entries in $R_{12}$. Milnor constructs a projective $R$-module $M\left(R_{1}^{n}, R_{2}^{n}, A\right)$ as follows.

As an abelian group,

$$
M\left(R_{1}^{n}, R_{2}^{n}, A\right)=\left\{(v, w) \in R_{1}^{n} \oplus R_{2}^{n} \mid A f(v)=g(w)\right\} .
$$

It becomes an $R=R_{1} \times_{R_{12}} R_{2}$ - module in the usual way. Now, since $g$ is surjective, there is a matrix $h_{A}$ with entries in $R_{2}$ such that $g\left(h_{A}\right)=A$, and there is a matrix $\tilde{h}_{A}$ with entries in $R_{2}$ such that $g\left(\tilde{h}_{A}\right)=A^{-1}$. Now, if

$$
X=X_{A}=\left[\begin{array}{cc}
1 & h_{A} \\
0 & 1
\end{array}\right]\left[\begin{array}{cc}
1 & 0 \\
-\tilde{h}_{A} & 1
\end{array}\right]\left[\begin{array}{cc}
1 & h_{A} \\
0 & 1
\end{array}\right]\left[\begin{array}{cc}
0 & -1 \\
1 & 0
\end{array}\right]
$$

then $X_{A}$ is an invertible $2 n \times 2 n$ matrix such that $g\left(X_{A}\right)=A \oplus A^{-1}$. 
If $S$ is a ring, we denote by $e_{i}^{S}$ the $i$-th standard basis vector of $S^{n}$. Furthermore, if $P$ is any direct summand of the $S$-module $S^{n}$, and if $h: S \rightarrow S^{\prime}$ is a ring homomorphism from $S$ to another ring $S^{\prime}$, we denote by $h_{*}(P)$ the $S^{\prime}$-span of the subgroup $h(P)$ of $\left(S^{\prime}\right)^{n}$ obtained by applying $h$ to the coordinates of the elements of $P$; it is a direct summand of the $S^{\prime}$-module $\left(S^{\prime}\right)^{n}$.

Now, as Milnor proves, the map $B: R^{n} \oplus R^{n} \rightarrow M\left(R_{1}^{n} \oplus R_{1}^{n}, R_{2}^{n} \oplus R_{2}^{n}, A \oplus A^{-1}\right)$ defined by

$$
B\left(e_{i}^{R}, 0\right)=\left(\left(e_{i}^{R_{1}}, 0\right), X_{A}\left(e_{i}^{R_{2}}, 0\right)\right)
$$

and

$$
B\left(0, e_{i}^{R}\right)=\left(\left(0, e_{i}^{R_{1}}\right), X_{A}\left(0, e_{i}^{R_{2}}\right)\right),
$$

for $1 \leqslant i \leqslant n$, is an isomorphism of $R$-modules.

Let $U: M\left(R_{1}^{n}, R_{2}^{n}, A\right) \rightarrow R^{n} \oplus R^{n}$ be the homomorphism of $R$-modules defined by

$$
U(v, w)=B^{-1}(v, 0, w, 0) .
$$

Let $P: R^{n} \oplus R^{n} \rightarrow M\left(R_{1}^{n}, R_{2}^{n}, A\right)$ be the homomorphism of $R$-modules defined by

$$
P=\pi_{1} \circ T \circ B
$$

where $T:\left(R_{1}^{n} \oplus R_{1}^{n}\right) \oplus\left(R_{2}^{n} \oplus R_{2}^{n}\right) \rightarrow\left(R_{1}^{n} \oplus R_{2}^{n}\right) \oplus\left(R_{1}^{n} \oplus R_{2}^{n}\right)$ is the isomorphism switching the middle two summands, and $\pi_{1}:\left(R_{1}^{n} \oplus R_{2}^{n}\right) \oplus\left(R_{1}^{n} \oplus R_{2}^{n}\right) \rightarrow\left(R_{1}^{n} \oplus R_{2}^{n}\right)$ is projection onto the first factor. Then, $P U=$ id and if $E=U P, E E=E$ and $M\left(R_{1}^{n}, R_{2}^{n}, A\right)$ is isomorphic to the direct summand $E\left(R^{n} \oplus R^{n}\right)$ of $R^{n} \oplus R^{n}$.

From now on, we set $M=E\left(R^{n} \oplus R^{n}\right)$.

Let $i_{1}: R \rightarrow R_{1}$ be the ring homomorphism $i_{1}\left(r_{1}, r_{2}\right)=r_{2}$, and let $i_{2}: R \rightarrow R_{2}$ be the ring homomorphism $i_{2}\left(r_{1}, r_{2}\right)=r_{2}$. (We are identifying $R$ with the subring $\left\{\left(r_{1}, r_{2}\right) \in R_{1} \times R_{2} \mid f\left(r_{1}\right)=g\left(r_{2}\right)\right\}$ of $R_{1} \times R_{2}$.)

A direct computation gives the following lemma.

\section{Lemma 6.3.}

(a) $\left(i_{1}\right)_{*}(M)=R_{1}^{n} \oplus 0 \subseteq R_{1}^{n} \oplus R_{1}^{n}$.

(b) $\left(i_{2}\right)_{*}(M)$ is equal to the $R_{2}$-submodule of $R_{2}^{n} \oplus R_{2}^{n}$ spanned by the $2 n$-vectors

$$
\left[\begin{array}{c}
e_{i}^{R_{2}} \\
0
\end{array}\right]-X^{-1}\left[\begin{array}{c}
0 \\
\left(1-\tilde{h}_{A} h_{A}\right) e_{i}^{R_{2}}
\end{array}\right]
$$

for $1 \leqslant i \leqslant n$ and

$$
X^{-1}\left[\begin{array}{c}
\left(h_{A} \tilde{h}_{A}-1\right) e_{i}^{R_{2}} \\
0
\end{array}\right]
$$

for $1 \leqslant i \leqslant n$.

(c) $f_{*}\left(\left(i_{1}\right)_{*}(M)\right)=\left(f i_{1}\right)_{*}(M)=\left(g i_{2}\right)_{*}(M)=g_{*}\left(\left(i_{2}\right)_{*}(M)\right)=R_{12}^{n} \oplus 0 \subseteq R_{12}^{n} \oplus R_{12}^{n}$. 
As a corollary, we have:

Corollary 6.4. The restriction of the $R_{2}$-module isomorphism defined by $X_{A}=X$ to the $R_{2}$-submodule $\left(i_{2}\right)_{*}(M)$ of $R_{2}^{n} \oplus R_{2}^{n}$ gives an isomorphism from $\left(i_{2}\right)_{*}(M)$ to the $R_{2}$-submodule $R_{2}^{n} \oplus 0$ of $R_{2}^{n} \oplus R_{2}^{n}$.

Milnor's homomorphism $\partial: K_{1}\left(R_{12}\right) \rightarrow K_{0}(R)$ may be described with the equation

$$
\partial\left(\left[R_{12}^{n}, A\right]\right)=\partial\left(\left[R_{12}^{n} \oplus 0, A \oplus 0\right]\right)=[M]-\left[R^{n} \oplus 0\right],
$$

where $R^{n} \oplus 0 \subseteq R^{n} \oplus R^{n}$.

Returning to the proof of Lemma 6.2, every element of $K_{1}\left(R_{12}\right)$ can be represented by $\left(R_{12}^{n}, A\right)$, where $A$ is an invertible $n \times n$-matrix with entries in $R_{12}$. There is the standard $R_{12}$-module inclusion $R_{12}^{n} \oplus 0 \subseteq R_{12}^{n} \oplus R_{12}^{n}$. Then,

$$
\begin{aligned}
\nu_{1 *}\left(\xi\left(\partial\left(\left[R_{12}^{n}, A\right]\right)\right)\right) & =\nu_{1 *}\left(\xi\left(\partial\left(\left[R_{12}^{n} \oplus 0, A \oplus 0\right]\right)\right)\right)=\nu_{1 *}\left(\xi\left([M]-\left[R^{n} \oplus 0\right]\right)\right) \\
& =\nu_{1 *}\left(t\left(\iota_{M}\right) t\left(\iota_{R^{n} \oplus 0}\right)^{-1}\right) \\
& =\left[\left(t\left(\iota_{i_{1 *}} M\right) t\left(\iota_{R_{1}^{n} \oplus 0}\right)^{-1}, t\left(\iota_{i_{2 *} M}\right) t\left(\iota_{R_{2}^{n} \oplus 0}\right)^{-1}\right)\right] .
\end{aligned}
$$

Using Lemma 6.3, we see that

$$
\begin{aligned}
{\left[\left(t\left(\iota_{i_{1 *} M}\right) t\left(\iota_{R_{1}^{n} \oplus 0}\right)^{-1}, t\left(\iota_{i_{2 *} M}\right) t\left(\iota_{R_{2}^{n} \oplus 0}\right)^{-1}\right)\right] } & =\left[\left(1, t\left(\iota_{i_{2 *} M}\right) t\left(\iota_{R_{2}^{n} \oplus 0}\right)^{-1}\right)\right] \\
& =\iota_{*}\left(\left[t\left(\iota_{i_{2 *} M}\right) t\left(\iota_{R_{2}^{n} \oplus 0}\right)^{-1}\right]\right) .
\end{aligned}
$$

However, consider the element $t\left(\iota_{i_{2 *} M}\right) t\left(\iota_{R_{2}^{n} \oplus 0}\right)^{-1}$ of $\left(G R_{2}\right)_{0}$. The element $x(A \oplus$ $0)$ of $\left(G R_{12}\right)_{1}$ represents the element $\left.\xi\left(\left[R_{12}^{n} \oplus 0, A \oplus 0\right)\right]\right)$. Let $Y$ be the isomorphism $\left.X\right|_{i_{2 *} M}: i_{2 *} M \rightarrow R_{2}^{n} \oplus 0$. Then, using the notation again from [6], we have an element $x(Y)=t\left(q_{i_{2 *} M} \mid Y\right) t\left(\iota_{i_{2 *} M} \mid Y\right)^{-1}$ of $\left(G R_{2}\right)_{1} \cap$ ker $d_{0}$; under the map $G R_{2} \rightarrow G R_{12}, x(Y)$ maps to $x(A \oplus 0)$ by Lemma $6.3(\mathrm{~b})$. Therefore, by definition and the results of $[\mathbf{6}]$,

$$
\delta_{2}\left(\xi\left(\left[R_{12}^{n}, A\right]\right)\right)=\delta_{2}([x(A)])=\delta_{2}([x(A \oplus 0)])=\left[d_{1}(x(Y))\right] .
$$

Also, by definition $([\mathbf{6}])$,

$$
\left[d_{1}(x(Y))\right]=\left[t\left(\iota_{\left.R_{2}^{n} \oplus 0\right)}\right)^{-1} t\left(\iota_{i_{2 *} M}\right)\right]=\left[t\left(\iota_{i_{2 *} M}\right) t\left(\iota_{\left.R_{2}^{n} \oplus 0\right)}\right)^{-1}\right] .
$$

Thus, we may conclude

Lemma 6.5. If, in the diagram $\mathfrak{R}$ above, $g$ is surjective and, in the diagram GR above, Gg is a fibration, then the homomorphism on zero-th homotopy groups induced by

$$
G R \rightarrow L \rightarrow \operatorname{Tot}(\mathcal{I}, G \Re)
$$

is an isomorphism.

On the other hand, the counterexample of Swan [18] shows that, without extra conditions on the maps $f, g$, this lemma cannot be extended to higher homotopy groups. 


\section{References}

[1] A.J. Berrick, An approach to algebraic K-theory, Research Notes in Mathematics 56, Pitman Advanced Publishing Program, Boston, 1982.

[2] C. Berger, Une groupoïde simplicial comme modèle de l'espace des chemins, Bull. Soc. Math. France 123 (1995), no. 1, 1-32.

[3] A.K. Bousfield, Homotopy spectral sequences and obstructions, Israel J. Math. 66 (1989), no. 1-3, 54-104.

[4] A.K. Bousfield and D.M. Kan, Homotopy limits, completions and localizations, Lecture Notes in Math. 304, Springer-Verlag, New York, 1987.

[5] A.K. Bousfield and D.M. Kan, A second quadrant homotopy spectral sequence, Trans. A.M.S. 177 (1973), 305-318.

[6] J. Duflot, Simplicial groups that are models for algebraic $K$-theory, Manuscripta Math. 113 (2004), no. 4, 423-470.

[7] J. Duflot and C.T. Marak, A filtration in algebraic K-theory, J. Pure Applied Algebra 151 (2000), no. 2, 135-162.

[8] P.G. Goerss and J.F. Jardine, Simplicial homotopy theory, Progress in Math. 174, Birkhäuser Verlag, Basel, 1999.

[9] A.R. Garzón and J.G. Miranda, Homotopy theory for truncated weak equivalences of simplicial groups, Math. Proc. Cambrdige Philos. Soc. 121 (1997), no. $1,51-74$.

[10] D.M. Kan. A combinatorial definition of homotopy groups, Annals of Math. 67 (1958), no. 2, 282-312.

[11] J.-L. Loday, $K$-théorie algébrique et représentations de groupes, Ann. Sci. École Norm. Sup. (4) 9 (1976), no. 3, 309-377.

[12] J.P. May, Simplicial objects in algebraic topology, Chicago Lecture Notes in Mathematics, The University of Chicago Press, Chicago, 1992.

[13] J. Milnor, Introduction to algebraic K-theory, Annals of Math. Studies 72, Princeton University Press, Princeton, 1971.

[14] D. Quillen, Higher algebraic K-theory, I, in Algebraic K-theory, I: Higher $K$-theories (Proc. Conf., Battelle Memorial Inst., Seattle, Wash., 1972), 85147, Lecture Notes in Math. 341, Springer-Verlag, New York, 1973.

[15] D. Quillen, Homotopical algebra, Lecture Notes in Math. 43, SpringerVerlag, New York, 1967.

[16] J. Rosenberg, Algebraic K-theory and its applications, Graduate Texts in Math. 147, Springer-Verlag, New York, 1994.

[17] V. Srinivas, Algebraic K-theory, Second Edition, Progress in Math. 90, Birkhäuser, Boston, 1996.

[18] R.G. Swan, Excision in algebraic K-theory, J. Pure Applied Algebra 1 (1971), no. 3, 221-252. 
[19] R.W. Thomason, Algebraic K-theory and étale cohomology, Ann. Sci. École Norm. Sup. (4) 18 (1985), no. 3, 437-552.

[20] R.W. Thomason, Le principe de scindage et l'inexistence d'une $K$-théorie de Milnor globale, Topology 31, no. 3 (1992), 571-588.

[21] J. Wu, On combinatorial descriptions of homotopy groups of certain spaces, Math. Proc. Cambridge Philos. Soc. 130 (2001), no. 3, 489-513.

Jeanne Duflot duflot@math.colostate.edu

Department of Mathematics, Colorado State University, Fort Collins, CO, 80523, USA 